\title{
A Comparison Study on Co- and Counterrotating Dual-Rotor System with Squeeze Film Dampers and Intermediate Bearing
}

\author{
Fei Wang, Gui-Huo Luo, Shu Yan, and Hai-Tao Cui \\ Jiangsu Province Key Laboratory of Aerospace Power System, Nanjing University of Aeronautics and Astronautics, \\ Nanjing 210016, China \\ Correspondence should be addressed to Gui-Huo Luo; ghluo@nuaa.edu.cn
}

Received 20 October 2016; Accepted 9 March 2017; Published 7 June 2017

Academic Editor: Chao Tao

Copyright (C) 2017 Fei Wang et al. This is an open access article distributed under the Creative Commons Attribution License, which permits unrestricted use, distribution, and reproduction in any medium, provided the original work is properly cited.

Nonlinear dynamic model of a coaxial rotor system was established with a method combining the finite element method and the fixed interface modal synthesis method. Then an implicit time domain method was presented to solve the nonlinear equations of motion; thus dynamic characteristics of the rotor system can be obtained. With nonlinear forces of squeeze film damper and intermediate bearing considered, nonlinear dynamic response characteristics of the co- and counterrotating coaxial rotor system under multiple unbalance forces were studied and compared in this work. It was found that the critical speeds of the corotating system were equal to or slightly higher than those of the counterrotating case due to the gyroscopic moments. The results showed that the unbalance excitation frequencies are dominant in the responses of the rotor system. Besides, due to coupling effect of the intermediate bearing some combinations of the unbalance excitation frequencies were also observed in the spectrogram but the combinations were different for co- and counterrotating cases. Stability and periodicity of the rotor system were investigated with bifurcation diagram, Poincare map, and phase diagram. It was found that the rotor system executes four-period quasi-periodic motion around critical speeds.

\section{Introduction}

In recent years, coaxial dual-rotor system has been applied to many aeroengines with two corotating or counterrotating rotors, such as RB211, GE120, and F119. The counterrotating technology is beneficial to improve the fuel consumption rate and thrust-weight ratio as well as reduce the gyroscopic torque of aeroengines [1]. Another technology that has been applied is the intermediate bearing which aims to further reduce the mass of the aeroengines. For most modern aeroengines, the squeeze film dampers (SFD) are commonly adopted to provide structural isolation and reduce the amplitude of rotor response. All these facts necessitate that more studies should be conducted to investigate the dynamic characteristics of dual-rotor systems with SFD and the intermediate bearing.

In 1975, Vance and Royal [2] have published an extensive discussion of the design and operational technological issues related to the intermediate bearings. Hibner [3] has applied the transfer matrix method to the multiple-shaft machines in order to compute the critical speeds and nonlinearly damped response. Gupta et al. [4] have presented a study on a counterrotating dual-rotor test rig with an intermediate bearing. The rotor system was also modeled with the transfer matrix method. Cross-excitation phenomena have been encountered. In 1996, Ferraris et al. [5] have analyzed the rotordynamics of a dual-shaft prop-fan aircraft engine with two rotors spin at equal speeds in opposite directions. A study of a twin-spool aircraft engine is also presented in the book of Lalanne and Ferraris [6]. In [7-9], Hu et al. have studied the dynamic characteristics of a counterrotating dual-rotor system with the transfer matrix method. The nonlinearity of the intermediate bearing was included. The finite element method has been applied in $[10,11]$ to obtain the dynamic characteristics of dual-rotor system with intermediate bearing. Fatigue life of two different configurations of the intermediate bearing were studied and compared by $\mathrm{Hu}$ et al. [12] in 2006. However, the intermediate bearing was 


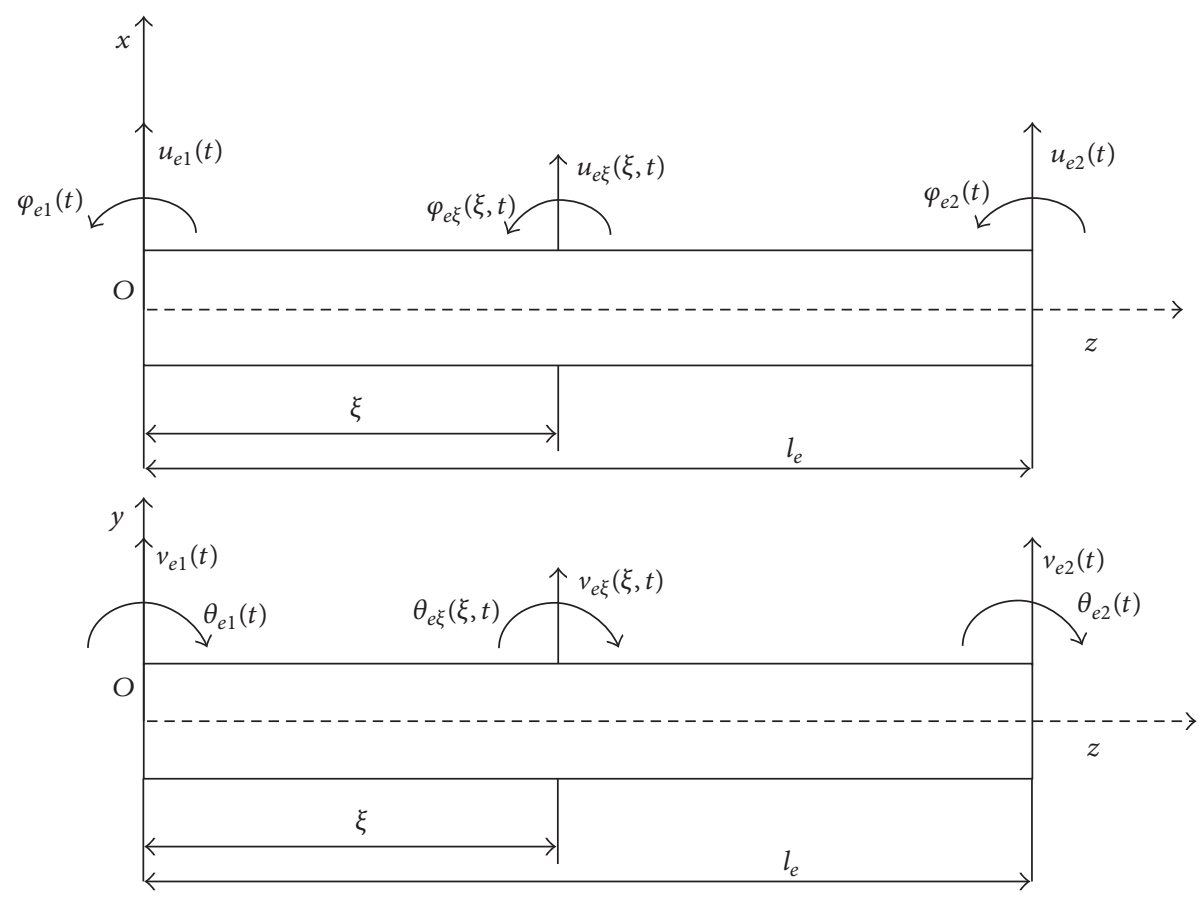

FIgURE 1: Diagram of beam element.

not coupled with the rotor system. From 2008 to 2011, Hai and Bonello [13-16] have conducted extensive investigations on computational method to obtain the unbalance response of the multispool whole aeroengine with squeeze film dampers.

The current study is on a co- and counterrotating dualrotor test rig that has been developed to study the dynamics of dual-rotor machines with intermediate bearings and squeeze film dampers. The purpose of this paper is to study the modeling method and nonlinear dynamic characteristics of the complex dual-rotor systems. In the second part of the paper, the modeling method and the numerical algorithm are presented. Then a description of the experimental apparatus is given in the next part. Finally, nonlinear dynamic characteristics of the co- and counterrotating test rig are studied and the numerical results are compared with the experimental results to validate the model established in this paper.

\section{Modeling and Numerical Algorithm}

2.1. Finite Element Formulation. Figure 1 shows the rotor element considered in this work. Each element consists of two nodes, with four degrees of freedom at each node. The nodal displacement vector can be described as

$$
\mathbf{q}_{\mathbf{e}}=\left[\begin{array}{llllllll}
u_{e 1} & v_{e 1} & \theta_{e 1} & \varphi_{e 1} & u_{e 2} & v_{e 2} & \theta_{e 2} & \varphi_{e 2}
\end{array}\right]^{T} .
$$

The displacement vector within the element can be interpolated as

$$
\begin{aligned}
& {\left[\begin{array}{c}
u_{e \xi}(\xi, t) \\
v_{e \xi}(\xi, t) \\
\theta_{e \xi}(\xi, t) \\
\varphi_{e \xi}(\xi, t)
\end{array}\right]} \\
& =\left[\begin{array}{cccccccc}
N_{1} & 0 & 0 & N_{2} & N_{3} & 0 & 0 & N_{4} \\
0 & N_{1} & -N_{2} & 0 & 0 & N_{3} & -N_{4} & 0 \\
0 & -\check{N}_{1} & \check{N}_{2} & 0 & 0 & -\check{N}_{3} & \check{N}_{4} & 0 \\
\check{N}_{1} & 0 & 0 & \check{N}_{2} & \check{N}_{3} & 0 & 0 & \check{N}_{4}
\end{array}\right] \mathbf{q}_{\mathbf{e}} .
\end{aligned}
$$

The shape functions in (2) are

$$
\begin{aligned}
& N_{1}=1-\frac{1}{l_{e}^{2}+12 g}\left(\frac{12 g}{l_{e}} \xi+3 \xi^{2}-\frac{2}{l_{e}} \xi^{3}\right), \\
& N_{2}=\frac{1}{l_{e}^{2}+12 g}\left(\left(l_{e}^{2}+6 g\right) \xi-\frac{2 l_{e}^{2}+6 g}{l_{e}} \xi^{2}+\xi^{3}\right), \\
& N_{3}=\frac{1}{l_{e}^{2}+12 g}\left(\frac{12 g}{l_{e}} \xi+3 \xi^{2}-\frac{2}{l_{e}} \xi^{3}\right), \\
& N_{4}=\frac{1}{l_{e}^{2}+12 g}\left(-6 g \xi-\frac{l_{e}^{2}-6 g}{l_{e}} \xi^{2}+\xi^{3}\right),
\end{aligned}
$$




$$
\begin{aligned}
& \check{N}_{1}=\frac{1}{l_{e}^{2}+12 g}\left(\frac{6}{l_{e}} \xi^{2}-6 \xi\right) \\
& \check{N}_{2}=\frac{1}{l_{e}^{2}+12 g}\left(l_{e}^{2}+12 g-\frac{4 l_{e}^{2}+12 g}{l_{e}} \xi+3 \xi^{2}\right) \\
& \check{N}_{3}=\frac{1}{l_{e}^{2}+12 g}\left(6 \xi-\frac{6}{l_{e}} \xi^{2}\right) \\
& \check{N}_{4}=\frac{1}{l_{e}^{2}+12 g}\left(\frac{12 g-2 l_{e}^{2}}{l_{e}} \xi+3 \xi^{2}\right) .
\end{aligned}
$$

In (3a) and (3b), $l_{e}$ is the element length and $g$ is formulated as given below:

$$
g=\frac{E_{e} I_{e}}{\kappa G_{e} A_{e}},
$$

where $E_{e}$ is Young's Modulus, $I_{e}$ is the area moment of inertia, $G_{e}$ is the shear modulus, and $A_{e}$ is the cross-sectional area. $\kappa$ denotes the shear coefficient, and for a hollow circular shaft section $[16,17]$

$$
\kappa=\frac{6(1+\mu)\left(1+\lambda^{2}\right)^{2}}{\left(7+12 \mu+4 \mu^{2}\right)\left(1+\lambda^{2}\right)^{2}+4\left(5+6 \mu+2 \mu^{2}\right) \lambda^{2}},
$$

where $\mu$ is Poisson's ratio and $\lambda$ is the ratio of the inner shaft radius to the outer shaft radius. Hence for a solid shaft $\lambda \rightarrow 0$.

With rotary inertia and shear effects considered, the kinetic energy and strain energy for a shaft element are

$$
\begin{aligned}
T_{e} & =\frac{1}{2} \int_{0}^{l_{e}}\left(\rho_{e} A_{e} \dot{u}_{e \xi}^{2}(\xi, t)+\rho_{e} I_{e} \dot{\varphi}_{e \xi}^{2}(\xi, t)\right) d \xi, \\
U_{e} & =\frac{1}{2} \int_{0}^{l_{e}}\left(\frac{\partial \varphi_{e \xi}(\xi, t)}{\partial \xi}\right)^{T} E_{e} I_{e}\left(\frac{\partial \varphi_{e \xi}(\xi, t)}{\partial \xi}\right) d \xi+\frac{1}{2} \\
& \cdot \kappa \int_{0}^{l_{e}} \gamma^{T} G_{e} A_{e} \gamma d \xi .
\end{aligned}
$$

And thus the element mass matrix is

$$
\begin{aligned}
& \mathbf{m}_{\mathbf{e}} \\
& =\rho_{e} A_{e} \int_{0}^{l_{e}}[\mathbf{N}]^{T}[\mathbf{N}] d \xi \\
& \quad+\rho_{e} I_{e} \int_{0}^{l_{e}}\left[g\left[\mathbf{N}^{\prime \prime \prime}\right]+\left[\mathbf{N}^{\prime}\right]\right]^{T}\left[g\left[\mathbf{N}^{\prime \prime \prime}\right]+\left[\mathbf{N}^{\prime}\right]\right] d \xi
\end{aligned}
$$

The element stiffness matrix is

$$
\begin{aligned}
\mathbf{k}_{\mathbf{e}}= & E_{e} I_{e} \int_{0}^{l_{e}}\left[\mathbf{N}^{\prime \prime}\right]^{T}\left[\mathbf{N}^{\prime \prime}\right] d \xi \\
& +E_{e} I_{e} g \int_{0}^{l_{e}}\left[\mathbf{N}^{\prime \prime \prime}\right]^{T}\left[\mathbf{N}^{\prime \prime \prime}\right] d \xi,
\end{aligned}
$$

where

$$
\begin{aligned}
& {\left[\mathbf{N}^{\prime}\right]=\frac{d\left[\begin{array}{llll}
N_{1} & N_{2} & N_{3} & N_{4}
\end{array}\right]}{d \xi},} \\
& {\left[\mathbf{N}^{\prime \prime}\right]=\frac{d^{2}\left[\begin{array}{llll}
N_{1} & N_{2} & N_{3} & N_{4}
\end{array}\right]}{d \xi^{2}},} \\
& {\left[\mathbf{N}^{\prime \prime \prime}\right]=\frac{d^{3}\left[\begin{array}{llll}
N_{1} & N_{2} & N_{3} & N_{4}
\end{array}\right]}{d \xi^{3}} .}
\end{aligned}
$$

The element gyroscopic matrix is

$$
\mathbf{g}_{\mathrm{e}}=-2 \rho_{e} I_{e} \int_{0}^{l_{e}}\left(\mathbf{B}_{2}^{\mathrm{T}} \mathbf{B}_{\mathbf{1}}-\mathbf{B}_{\mathbf{1}}^{\mathrm{T}} \mathbf{B}_{\mathbf{2}}\right) d \xi
$$

where

$$
\left[\begin{array}{l}
\mathbf{B}_{1} \\
\mathbf{B}_{2}
\end{array}\right]=\left[\begin{array}{cccccccc}
0 & -\check{N}_{1} & \check{N}_{2} & 0 & 0 & -\check{N}_{3} & \check{N}_{4} & 0 \\
\check{N}_{1} & 0 & 0 & \check{N}_{2} & \check{N}_{3} & 0 & 0 & \check{N}_{4}
\end{array}\right] .
$$

For disk element, the mass and gyroscopic matrices are

$$
\begin{aligned}
\mathbf{m}_{\mathbf{e}}^{\mathbf{d}} & =\left[\begin{array}{cccc}
m_{d}^{i} & 0 & 0 & 0 \\
0 & m_{d}^{i} & 0 & 0 \\
0 & 0 & I_{d}^{i} & 0 \\
0 & 0 & 0 & I_{d}^{i}
\end{array}\right], \\
\mathbf{g}_{\mathbf{e}}^{\mathbf{d}} & =\left[\begin{array}{llll}
0 & 0 & 0 & 0 \\
0 & 0 & 0 & 0 \\
0 & 0 & 0 & I_{p}^{i} \\
0 & 0 & -I_{p}^{i} & 0
\end{array}\right],
\end{aligned}
$$

where $m_{d}^{i}$, $I_{d}^{i}$, and $I_{p}^{i}$ are the mass, diametral moment of inertia, and polar moment of inertia of the disk, respectively.

2.2. Nonlinear Forces of the Supports. For squeeze film dampers analyzed in this work, based on the short bearing assumption and the Reynolds boundary conditions, nonlinear forces of the SFD can be expressed as [17]

$$
\begin{aligned}
f_{x}^{\mathrm{sfd}} & =-\frac{\mu_{s} R L^{3}}{c^{2}\left(x^{2}+y^{2}\right)^{1 / 2}}\left[x\left(\dot{\varepsilon} I_{2}+\varepsilon \dot{\psi} I_{1}\right)\right. \\
& \left.-y\left(\dot{\varepsilon} I_{1}+\varepsilon \dot{\psi} I_{3}\right)\right], \\
f_{y}^{\mathrm{sfd}} & =-\frac{\mu_{s} R L^{3}}{c^{2}\left(x^{2}+y^{2}\right)^{1 / 2}}\left[y\left(\dot{\varepsilon} I_{2}+\varepsilon \dot{\psi} I_{1}\right)\right. \\
& \left.+x\left(\dot{\varepsilon} I_{1}+\varepsilon \dot{\psi} I_{3}\right)\right], \\
\varepsilon & =\frac{\sqrt{x^{2}+y^{2}}}{c}
\end{aligned}
$$




$$
\begin{aligned}
& \dot{\varepsilon}=\frac{x \dot{x}+y \dot{y}}{c \sqrt{x^{2}+y^{2}}}, \\
& \dot{\psi}=\frac{y \dot{x}-x \dot{y}}{x^{2}+y^{2}}, \\
& \tan \psi=\frac{x}{y} .
\end{aligned}
$$

$x$ and $y$ are the horizontal and vertical displacements of the journal. $I_{j}(j=1,2,3)$ are Sommerfeld integrals. $R$ and $L$ are radius and length of the SFD, respectively. $\mu_{s}$ and $c$ denote the dynamic viscosity of the film and the radial clearance of the SFD.

As for the rolling ball bearing, based on pure rolling assumption and the hertz contact theory, nonlinear force of the rolling ball bearing [18] is

$$
\begin{aligned}
f_{x}^{b} & =k_{n} \sum_{j=1}^{N_{b}} u_{\theta j}^{\xi} H\left(u_{\theta j}\right) \sin \theta_{j}, \\
f_{y}^{b} & =k_{n} \sum_{j=1}^{N_{b}} u_{\theta j}^{\xi} H\left(u_{\theta j}\right) \cos \theta_{j}, \\
\theta_{j} & =\frac{2 \pi(j-1)}{N_{b}}+\omega_{c} t, \\
u_{\theta j} & =\left(x^{\text {ir }}-x^{\text {or }}\right) \cos \theta_{j}+\left(y^{\text {ir }}-y^{\text {or }}\right) \sin \theta_{j}-\frac{\gamma}{2}, \\
H\left(u_{\theta j}\right) & =\left\{\begin{array}{l}
0, \quad u_{\theta j} \leq 0, \\
u_{\theta j}, \quad u_{\theta j}>0,
\end{array}\right. \\
\omega_{c} & =\frac{\omega_{\text {in }} r+\omega_{\text {out }} R}{R+r},
\end{aligned}
$$

where the superscripts ir and or in (14c) denote inner ring and outer ring of the bearing. $k_{n}$ in (14a) is the contact stiffness between rollers and rings. $N_{b}$ represent number of the rollers. $\xi=3 / 2$ for the rolling ball bearing used in the intermediate support. $\theta_{j}$ is the rotation angle of the $j$ th roller at time $t$. $\gamma$ and $\omega_{c}$ are bearing radial clearance and rotational speed of the bearing retainer, respectively. $u_{\theta j}$ is the elastic radial deformation of the $j$ th roller. $r$ and $R$ represent radius of the inner and outer ring. $\omega_{\text {in }}$ and $\omega_{\text {out }}$ are the rotational speeds of the inner and outer rings.

2.3. Equations of Motion and Numerical Algorithm. Equation of motion of a nonlinear rotor system can be written as

$$
\mathbf{M} \ddot{\mathbf{u}}+\mathbf{G} \dot{\mathbf{u}}+\mathbf{K u}=\mathbf{F}^{\text {nonl }}+\mathbf{F}^{\mathbf{u n b}} .
$$

$\mathbf{F}^{\mathbf{u n b}}$ is the unbalance force vector. $\mathbf{F}^{\mathbf{n o n l}}$ is the nonlinear forces exerted by the SFDs and rolling bearing in this work. $\mathbf{M}, \mathbf{K}$, and $\mathbf{G}$ can be obtained quite readily with elements formulated in Section 2.1.

Mathematically, the model is a set of nonlinear secondorder differential equations, the computational efficiency of which depends on the numerical methods applied and the total DOFs of the rotor system. To combine accuracy of the finite element model and computational efficiency, the fixed interface modal synthesis is applied to reduce dimension of the mathematical model and thus the computational effort.

Equation (15) can be rewritten as

$$
\begin{aligned}
& {\left[\begin{array}{ll}
M_{I I} & M_{I J} \\
M_{I I} & M_{I J}
\end{array}\right]\left[\begin{array}{l}
\ddot{\mathbf{u}}_{I} \\
\ddot{\mathbf{u}}_{\mathrm{J}}
\end{array}\right]+\left[\begin{array}{ll}
\mathrm{G}_{\mathrm{II}} & \mathrm{G}_{\mathrm{IJ}} \\
\mathrm{G}_{\mathrm{II}} & \mathrm{G}_{\mathrm{IJ}}
\end{array}\right]\left[\begin{array}{c}
\dot{\mathrm{u}}_{\mathrm{I}} \\
\dot{\mathrm{u}}_{\mathrm{J}}
\end{array}\right]} \\
& +\left[\begin{array}{cc}
\mathrm{K}_{\mathrm{II}} & \mathrm{K}_{\mathrm{IJ}} \\
\mathrm{K}_{\mathrm{JI}} & \mathrm{K}_{\mathrm{JJ}}
\end{array}\right]\left[\begin{array}{c}
\mathbf{u}_{\mathrm{I}} \\
\mathbf{u}_{\mathrm{J}}
\end{array}\right]=\left[\begin{array}{c}
\mathrm{F}_{\mathrm{I}}^{\mathrm{unb}} \\
0
\end{array}\right]+\left[\begin{array}{c}
0 \\
\mathrm{~F}_{\mathrm{J}}^{\text {nonl }}
\end{array}\right],
\end{aligned}
$$

where $\mathbf{u}_{\mathbf{I}}$ can be interpreted as linear DOFs, with only unbalance forces considered, while $\mathbf{u}_{\mathrm{J}}$ represents the interface DOFs or nonlinear DOFs in this work, including all the nodes with nonlinear forces considered.

According to fixed interface modal synthesis method, transformation between physical and modal coordinates is

$$
\left[\begin{array}{c}
\mathbf{u}_{\mathrm{I}} \\
\mathbf{u}_{\mathrm{J}}
\end{array}\right]=\left[\begin{array}{cc}
\phi_{\mathrm{k}} & \phi_{\mathrm{c}} \\
\mathbf{0} & \mathrm{I}
\end{array}\right]\left[\begin{array}{l}
\mathbf{q}_{\mathrm{k}} \\
\mathbf{u}_{\mathrm{J}}
\end{array}\right]=\mathrm{T}\left[\begin{array}{l}
\mathbf{q}_{\mathrm{k}} \\
\mathbf{u}_{\mathrm{J}}
\end{array}\right],
$$

where $\phi_{\mathbf{k}}$ is the mass normalized normal mode matrix, $\phi_{\mathbf{c}}$ is the mass normalized constrained mode, $\mathbf{I}$ is unity matrix, $\mathbf{q}_{\mathbf{k}}$ is the normal mode coordinates, and $\mathbf{T}$ is the transformation matrix. $\phi_{c}$ is given as below:

$$
\phi_{\mathrm{c}}=-\left(\mathbf{K}_{\mathrm{II}}\right)^{-1} \mathbf{K}_{\mathrm{IJ}} \text {. }
$$

In (16), let $\mathbf{u}_{\mathbf{J}}=\mathbf{0}$ and neglecting the gyroscopic effects gives

$$
\mathbf{M}_{\mathrm{II}} \ddot{\mathbf{u}}_{\mathrm{I}}+\mathrm{K}_{\mathrm{II}} \mathbf{u}_{\mathrm{I}}=\mathbf{0} \text {. }
$$

Solving the eigenvalue problem corresponding to (17c) gives the mass normalized normal mode matrix $\phi_{\mathbf{k}}$ and the modal frequency $\boldsymbol{\Omega}_{\mathbf{k}}$.

Substitute (17a) into (16) and premultiply with $\mathbf{T}^{\mathbf{T}}$ to obtain the reduced equations of motion:

$$
\begin{gathered}
{\left[\begin{array}{cc}
\mathrm{I} & \overline{\mathbf{M}}_{\mathrm{IJ}} \\
\overline{\mathbf{M}}_{\mathrm{II}} & \overline{\mathbf{M}}_{\mathrm{JJ}}
\end{array}\right]\left[\begin{array}{c}
\ddot{\mathbf{q}}_{\mathrm{k}} \\
\ddot{\mathbf{u}}_{\mathrm{J}}
\end{array}\right]+\left[\begin{array}{ll}
\overline{\mathrm{G}}_{\mathrm{II}} & \overline{\mathrm{G}}_{\mathrm{IJ}} \\
\overline{\mathrm{G}}_{\mathrm{JI}} & \overline{\mathbf{G}}_{\mathrm{JJ}}
\end{array}\right]\left[\begin{array}{l}
\dot{\mathbf{q}}_{\mathrm{k}} \\
\dot{\mathbf{u}}_{\mathrm{J}}
\end{array}\right]} \\
+\left[\begin{array}{cc}
\overline{\mathbf{K}}_{\mathrm{II}} & 0 \\
0 & \overline{\mathbf{K}}_{\mathrm{JJ}}
\end{array}\right]\left[\begin{array}{l}
\mathbf{q}_{\mathrm{k}} \\
\mathbf{u}_{\mathrm{J}}
\end{array}\right]=\left[\begin{array}{c}
\phi_{\mathrm{k}}^{\mathrm{T}} \mathrm{F}_{\mathrm{I}}^{\mathrm{unb}} \\
\phi_{\mathrm{c}}^{\mathrm{T}} \mathrm{F}_{\mathrm{I}}^{\mathrm{unb}}
\end{array}\right]+\left[\begin{array}{c}
0 \\
\mathrm{~F}_{\mathrm{J}}^{\text {nonl }}
\end{array}\right],
\end{gathered}
$$

where

$$
\begin{aligned}
& \overline{\mathbf{M}}_{\mathrm{JJ}}=\mathrm{M}_{\mathrm{JJ}}+\mathrm{M}_{\mathrm{JI}} \phi_{\mathrm{c}}+\phi_{\mathrm{c}}^{\mathrm{T}}\left(\mathrm{M}_{\mathrm{II}} \phi_{\mathrm{c}}+\mathrm{M}_{\mathrm{IJ}}\right), \\
& \overline{\mathbf{M}}_{\mathrm{IJ}}=\overline{\mathbf{M}}_{\mathrm{JI}}=\phi_{\mathrm{k}}^{\mathrm{T}}\left(\mathrm{M}_{\mathrm{II}} \phi_{\mathrm{c}}+\mathrm{M}_{\mathrm{IJ}}\right) \text {, } \\
& \overline{\mathrm{G}}_{\mathrm{II}}=\phi_{\mathrm{k}}^{\mathrm{T}} \mathrm{G}_{\mathrm{II}} \phi_{\mathrm{k}} \\
& \overline{\mathrm{G}}_{\mathrm{IJ}}=\mathrm{G}_{\mathrm{JJ}}+\mathrm{G}_{\mathrm{JI}} \phi_{\mathrm{c}}+\phi_{\mathrm{c}}^{\mathrm{T}}\left(\mathrm{G}_{\mathrm{II}} \phi_{\mathrm{c}}+\mathrm{G}_{\mathrm{IJ}}\right)+\mathrm{c}_{\mathrm{JJ}} \text {, } \\
& \overline{\mathrm{G}}_{\mathrm{IJ}}=\overline{\mathrm{G}}_{\mathrm{II}}=\phi_{\mathrm{k}}^{\mathrm{T}} \mathrm{G}_{\mathrm{II}} \phi_{\mathrm{c}}+\phi_{\mathrm{k}}^{\mathrm{T}} \mathrm{G}_{\mathrm{IJ}} \text {, } \\
& \overline{\mathbf{K}}_{\mathrm{II}}=\operatorname{diag}\left(\Omega_{r}^{2}\right), \quad 1 \leq r \leq n, \\
& \overline{\mathbf{K}}_{\mathrm{JJ}}=\mathbf{K}_{\mathrm{IJ}}+\mathbf{K}_{\mathrm{JI}} \phi_{\mathrm{c}}+\mathbf{k}_{\mathrm{JJ}} \cdot
\end{aligned}
$$


$\Omega_{r}$ is the $r$ th modal frequency obtained from (17c). $\mathrm{F}_{\mathrm{I}}^{\mathrm{unb}}$ is the unbalance force acting on linear DOFs. $\mathbf{F}_{\mathbf{J}}^{\text {nonl }}$ is the nonlinear force acting on nonlinear DOFs.

And

$$
\mathbf{F}_{\mathrm{J}}^{\text {nonl }}=-\mathbf{k}_{\mathrm{JJ}}+\left[\begin{array}{c}
\mathbf{F}_{\mathrm{J}}^{\mathrm{SFD}} \\
\mathbf{F}_{\mathrm{J}}^{\mathrm{B}}
\end{array}\right],
$$

where $\mathbf{F}_{\mathbf{J}}^{\mathrm{SFD}}=\left[\begin{array}{lllll}f_{1 x}^{\mathrm{sfd}} & f_{1 y}^{\mathrm{sfd}} & \cdots & f_{N x}^{\mathrm{sfd}} & f_{N y}^{\mathrm{sfd}}\end{array}\right]$ is the nonlinear force vector exerted by SFDs which can be calculated with (13a), (13b), (13c), (13d), and (13e). $\mathbf{F}_{\mathbf{J}}^{\mathbf{B}}=\left[\begin{array}{llll}f_{x}^{b} & f_{y}^{b} & -f_{x}^{b} & -f_{y}^{b}\end{array}\right]$ is the nonlinear force vector exerted by rolling bearing which can be calculated with (14a), (14b), (14c), (14d), and (14e). $\mathbf{k}_{\mathrm{JJ}}$ and $\boldsymbol{c}_{\mathrm{JJ}}$ are given below:

$$
\begin{aligned}
& \mathbf{k}_{\mathrm{JJ}}=\left[\begin{array}{ll}
\mathbf{k}_{\mathbf{J}}^{\mathbf{B}} \mathbf{0} \\
\mathbf{0} & \mathbf{0}
\end{array}\right], \\
& \mathbf{c}_{\mathrm{JJ}}=\left[\begin{array}{ll}
\mathbf{c}_{\mathbf{J}}^{\mathbf{B}} & \mathbf{0} \\
\mathbf{0} & \mathbf{0}
\end{array}\right], \\
& \mathbf{k}_{\mathbf{J}}^{\mathbf{B}}=\operatorname{diag}\left(\mathbf{k}_{\mathbf{J i}}^{\mathbf{B}}\right), \quad 1 \leq i \leq N, \\
& \mathbf{c}_{\mathbf{J}}^{\mathbf{B}}=\operatorname{diag}\left(\mathbf{c}_{\mathrm{Ji}}^{\mathbf{B}}\right), \quad 1 \leq i \leq N, \\
& \mathbf{k}_{\mathrm{Ji}}^{\mathbf{B}}=\left[\begin{array}{cc}
k_{J i} & 0 \\
0 & k_{J i}
\end{array}\right], \\
& \mathbf{c}_{J \mathbf{i}}^{\mathbf{B}}=\left[\begin{array}{cc}
c_{J i} & 0 \\
0 & c_{J i}
\end{array}\right] .
\end{aligned}
$$

$k_{J i}$ and $c_{J i}$ are the stiffness and damping coefficients of the elastic support, respectively. $N$ is the number of elastic supports.

Rearranging (18a) and (18b) gives

$$
\begin{aligned}
& \ddot{\mathbf{q}}_{\mathrm{k}}+\overline{\mathrm{G}}_{\mathrm{II}} \dot{\mathbf{q}}_{\mathrm{k}}+\overline{\mathbf{K}}_{\mathrm{II}} \mathbf{q}_{\mathrm{k}}=\phi_{\mathrm{k}}^{\mathrm{T}} \mathrm{F}_{\mathrm{I}}^{\mathrm{unb}}-\overline{\mathbf{M}}_{\mathrm{IJ}} \ddot{\mathbf{u}}_{\mathrm{J}}-\overline{\mathrm{G}}_{\mathrm{IJ}} \dot{\mathbf{u}}_{\mathrm{I}}, \\
& \overline{\mathbf{M}}_{\mathrm{IJ}} \ddot{\mathbf{u}}_{\mathrm{J}}+\overline{\mathrm{G}}_{\mathrm{JJ}} \dot{\mathbf{u}}_{\mathrm{J}}+\overline{\mathbf{K}}_{\mathrm{IJ}} \mathbf{u}_{\mathrm{J}} \\
& =\mathrm{F}_{\mathrm{J}}^{\mathrm{nonl}}+\phi_{\mathrm{c}}^{\mathrm{T}} \mathrm{F}_{\mathrm{I}}^{\mathrm{unb}}-\overline{\mathbf{M}}_{\mathrm{JI}} \ddot{\mathbf{q}}_{\mathrm{k}}-\overline{\mathrm{G}}_{\mathrm{II}} \dot{\mathbf{q}}_{\mathrm{k}} .
\end{aligned}
$$

In (21a) and (21b), the vectors $\mathbf{q}_{\mathbf{k}}$ and $\mathbf{u}_{\boldsymbol{J}}$ can be interpreted as linear and nonlinear DOFs of the reduced system, respectively. Obviously, there is no nonlinear force acting on the linear DOFs. Thus, the explicit Newmark-beta method applies to (21a) while implicit Newmark-beta method applies to (21b).
According to assumptions of Newmark-beta method, in time interval $\left[\begin{array}{ll}t_{n} & t_{n+1}\end{array}\right]$ is

$$
\begin{aligned}
{\left[\begin{array}{c}
\dot{\mathbf{q}}_{\mathbf{k}}^{\mathbf{n}+1} \\
\dot{\mathbf{u}}_{\mathrm{J}}^{\mathbf{n}+1}
\end{array}\right]=} & {\left[\begin{array}{c}
\dot{\mathbf{q}}_{\mathbf{k}}^{\mathbf{n}} \\
\dot{\mathbf{u}}_{\mathrm{J}}^{\mathbf{n}}
\end{array}\right]+\left\{(1-\beta)\left[\begin{array}{c}
\ddot{\mathbf{q}}_{\mathbf{k}}^{\mathbf{n}} \\
\ddot{\mathbf{u}}_{\mathrm{J}}^{\mathbf{n}}
\end{array}\right]+\beta\left[\begin{array}{c}
\ddot{\mathbf{q}}_{\mathbf{k}}^{\mathbf{n}+1} \\
\ddot{\mathbf{u}}_{\mathrm{J}}^{\mathbf{n}+1}
\end{array}\right]\right\} \Delta t, } \\
{\left[\begin{array}{c}
\mathbf{q}_{\mathbf{k}}^{\mathbf{n}+1} \\
\mathbf{u}_{\mathrm{J}}^{\mathbf{n}+1}
\end{array}\right]=} & {\left[\begin{array}{c}
\mathbf{q}_{\mathbf{k}}^{\mathbf{n}} \\
\mathbf{u}_{\mathrm{J}}^{\mathbf{n}}
\end{array}\right]+\left[\begin{array}{c}
\dot{\mathbf{q}}_{\mathbf{k}}^{\mathbf{n}} \\
\dot{\mathbf{u}}_{\mathrm{J}}^{\mathbf{n}}
\end{array}\right] \Delta t } \\
& +\left\{(0.5-\alpha)\left[\begin{array}{c}
\ddot{\mathbf{q}}_{\mathbf{k}}^{\mathbf{n}} \\
\ddot{\mathbf{u}}_{\mathrm{J}}^{\mathbf{n}}
\end{array}\right]+\alpha\left[\begin{array}{c}
\ddot{\mathbf{q}}_{\mathbf{k}}^{\mathbf{n}+1} \\
\ddot{\mathbf{u}}_{\mathrm{J}}^{\mathbf{n}+1}
\end{array}\right]\right\} \Delta t^{2},
\end{aligned}
$$

where $t_{n+1}=t_{n}+\Delta t$ and $\Delta t$ is the time increment. The superscripts $n$ and $n+1$ denote $t_{n}$ and $t_{n+1}$. (22b):

The following equations can be obtained from (22a) and

$$
\begin{aligned}
& \ddot{\mathbf{q}}_{\mathbf{k}}^{\mathbf{n}+1}=a \mathbf{q}_{\mathbf{k}}^{\mathbf{n}+1}-\mathbf{A}_{\mathbf{q}}^{\mathbf{n}}, \\
& \dot{\mathbf{q}}_{\mathbf{k}}^{\mathbf{n}+1}=b \mathbf{q}_{\mathbf{k}}^{\mathbf{n}+1}-\mathbf{B}_{\mathbf{q}}^{\mathbf{n}}, \\
& \ddot{\mathbf{u}}_{\mathrm{J}}^{\mathbf{n}+1}=a \mathbf{u}_{\mathrm{J}}^{\mathbf{n}+1}-\mathbf{A}_{\mathrm{J}}^{\mathbf{n}}, \\
& \dot{\mathbf{u}}_{\mathrm{J}}^{\mathbf{n}+1}=b \mathbf{u}_{\mathrm{J}}^{\mathbf{n}+1}-\mathbf{B}_{\mathrm{J}}^{\mathbf{n}},
\end{aligned}
$$

with

$$
\begin{aligned}
\mathbf{A}_{\mathbf{q}}^{\mathbf{n}} & =\frac{1}{\alpha \Delta t^{2}} \mathbf{q}_{\mathbf{k}}^{\mathbf{n}}+\frac{1}{\alpha \Delta t} \dot{\mathbf{q}}_{\mathbf{k}}^{\mathbf{n}}+\left(\frac{1}{2 \alpha}-1\right) \ddot{\mathbf{q}}_{\mathbf{k}}^{\mathbf{n}}, \\
\mathbf{A}_{\mathbf{J}}^{\mathbf{n}} & =\frac{1}{\alpha \Delta t^{2}} \mathbf{u}_{\mathbf{k}}^{\mathbf{n}}+\frac{1}{\alpha \Delta t} \dot{\mathbf{u}}_{\mathbf{k}}^{\mathbf{n}}+\left(\frac{1}{2 \alpha}-1\right) \ddot{\mathbf{u}}_{\mathbf{k}}^{\mathbf{n}}, \\
\mathbf{B}_{\mathbf{q}}^{\mathbf{n}} & =\frac{\beta}{\alpha \Delta t} \mathbf{q}_{\mathbf{k}}^{\mathbf{n}}+\left(\frac{\beta}{\alpha}-1\right) \dot{\mathbf{q}}_{\mathbf{k}}^{\mathbf{n}}+\left(\frac{\beta}{\alpha}-2\right) \ddot{\mathbf{q}}_{\mathbf{k}}^{\mathbf{n}}, \\
\mathbf{B}_{\mathbf{J}}^{\mathbf{n}} & =\frac{\beta}{\alpha \Delta t} \mathbf{u}_{\mathbf{k}}^{\mathbf{n}}+\left(\frac{\beta}{\alpha}-1\right) \dot{\mathbf{u}}_{\mathbf{k}}^{\mathbf{n}}+\left(\frac{\beta}{\alpha}-2\right) \ddot{\mathbf{u}}_{\mathbf{k}}^{\mathbf{n}}, \\
a & =\frac{1}{\alpha \Delta t^{2}}, \\
b & =\frac{\beta}{\alpha \Delta t} .
\end{aligned}
$$

Substituting (22a) and (22b)-(25) into (21a) and (21b) yields

$$
\begin{aligned}
\mathbf{q}_{k}^{n+1} & =S_{q}^{-1}\left(\phi_{k}^{T} F_{I}^{u n b n+1}-V_{q}^{u_{J}^{n+1}}+W_{q}\right), \\
\left(S_{J}\right. & \left.-V_{J} S_{q}^{-1} V_{q}\right) u_{J}^{n+1} \\
= & F_{J}^{n o n l} n^{n+1}+\left(\phi_{c}^{T}-V_{J} S_{q}^{-1} \phi_{k}^{T}\right) F_{I}^{u n b n+1} \\
& -V_{J} S_{q}^{-1} W_{q}+W_{J},
\end{aligned}
$$




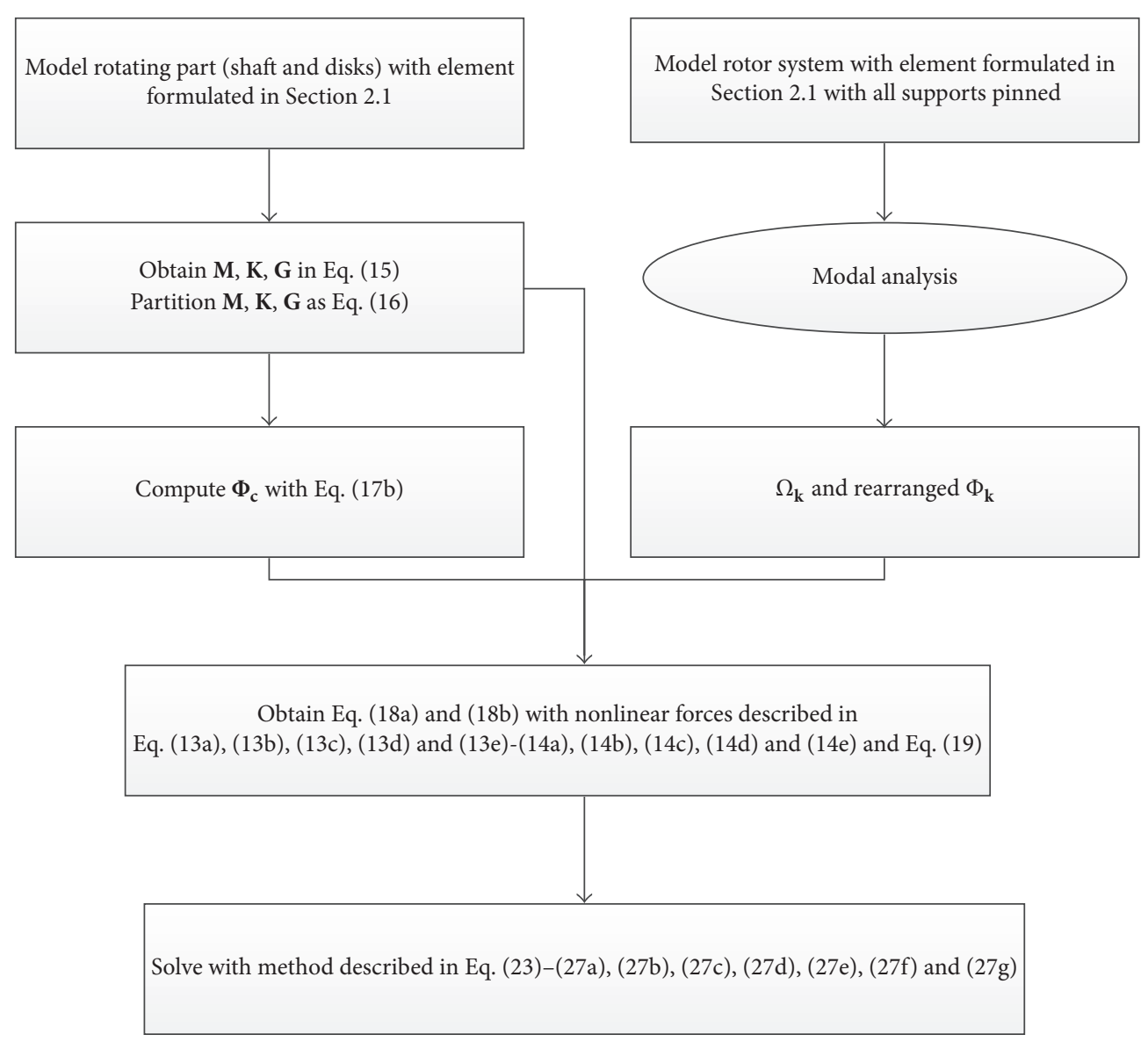

FIgURE 2: Flowchart of modeling and solving.

with

$$
\begin{aligned}
\mathbf{W}_{\mathbf{q}} & =\overline{\mathbf{M}}_{\mathrm{II}} \mathbf{A}_{\mathbf{q}}^{\mathbf{n}}+\overline{\mathbf{G}}_{\mathrm{II}} \mathbf{B}_{\mathbf{q}}^{\mathbf{n}}+\overline{\mathbf{M}}_{\mathrm{IJ}} \mathbf{A}_{\mathrm{J}}^{\mathbf{n}}+\overline{\mathbf{G}}_{\mathrm{IJ}} \mathbf{B}_{\mathrm{J}}^{\mathbf{n}}, \\
\mathbf{W}_{\mathrm{J}} & =\overline{\mathbf{M}}_{\mathrm{JJ}} \mathbf{A}_{\mathrm{J}}^{\mathbf{n}}+\overline{\mathbf{G}}_{\mathrm{JJ}} \mathbf{B}_{\mathrm{J}}^{\mathbf{n}}+\overline{\mathbf{M}}_{\mathrm{JI}} \mathbf{A}_{\mathbf{q}}^{\mathbf{n}}+\overline{\mathbf{G}}_{\mathrm{JI}} \mathbf{B}_{\mathbf{q}}^{\mathbf{n}}, \\
\mathbf{S}_{\mathbf{q}} & =a \overline{\mathbf{M}}_{\mathrm{II}}+b \overline{\mathbf{G}}_{\mathrm{II}}+\overline{\mathbf{K}}_{\mathrm{II}}, \\
\mathbf{S}_{\mathrm{J}} & =a \overline{\mathbf{M}}_{\mathrm{JJ}}+b \overline{\mathbf{G}}_{\mathrm{JJ}}+\overline{\mathbf{K}}_{\mathrm{JJ}}, \\
\mathbf{V}_{\mathbf{q}} & =a \overline{\mathbf{M}}_{\mathrm{IJ}}+b \overline{\mathbf{G}}_{\mathrm{IJ}}, \\
\mathbf{V}_{\mathbf{J}} & =a \overline{\mathbf{M}}_{\mathrm{JI}}+b \overline{\mathbf{G}}_{\mathrm{JI}}, \\
\mathbf{F}_{\mathbf{J}}^{\mathbf{n o n l} \mathbf{n}+\mathbf{1}} & =\mathbf{F}_{\mathbf{J}}^{\mathbf{n o n l} \mathbf{n}}\left(\mathbf{u}_{\mathbf{J}}^{\mathbf{n}+\mathbf{1}}, \dot{\mathbf{u}}_{\mathrm{J}}^{\mathbf{n}+\mathbf{1}}\right) .
\end{aligned}
$$

Use (24) to substitute for $\dot{\mathbf{u}}_{\mathbf{J}}^{\mathbf{n}+\mathbf{1}}$ in (27g) and substitute the new (27g) into (26b) to yield nonlinear equations of $\mathbf{u}_{\mathrm{J}}^{\mathbf{n}+\mathbf{1}}$. After solving (26b) for $\mathbf{u}_{\mathbf{J}}^{\mathbf{n}+\mathbf{1}}$ with numerical algorithms, $\mathbf{q}_{\mathbf{k}}^{\mathbf{n}+\mathbf{1}}$ can be obtained with $\mathbf{u}_{\mathbf{J}}^{\mathbf{n}+\mathbf{1}}$ substituted into (26a). Thus $\dot{\mathbf{u}}_{\mathbf{J}}^{\mathbf{n}+\mathbf{1}}, \ddot{\mathbf{u}}_{\mathrm{J}}^{\mathbf{n}+\mathbf{1}}$, $\dot{\mathbf{q}}_{\mathbf{k}}^{\mathbf{n}+\mathbf{1}}$, and $\ddot{\mathbf{q}}_{\mathbf{k}}^{\mathbf{n}+\mathbf{1}}$ can be solved from (23) and (24). The process continues to repeat and move forward to find $\mathbf{u}_{\mathrm{J}}^{\mathbf{n}+2}$ and so on. When the iteration is over, the nonlinear response in physical coordinate system can be obtained from (17a).
Computational efficiency of solving (21a) and (21b) depends on solving (21b) while dimensions of (21b) depend on DOFs with nonlinear forces considered. Thus the computational efficiency can be greatly improved with the modeling technique and solving method described in this work.

To summarize, nonlinear model of the rotor system is established with finite element method and fixed interface modal synthesis method; subsequently an implicit timedomain method based on Newmark-beta method is applied to solve the equations of motion of the reduced system; thus dynamic characteristics can be obtained. Flowchart of the modeling and solving method is shown in Figure 2.

\section{Test Rig Description}

The coaxial test rig studied in this paper is presented in Figure 3.

The stationary coordinate system in Figure 3 consists of three mutually perpendicular axes, $o x, o y$, and $o z$, intersecting at the point $o$ and axis of the rotor coincides with axis $o z$. The axes $o x$ and $o z$ are horizontal, and $o y$ is vertical.

The rotor system consists of two shafts disposed along the same axis $o z$, connected by an intermediate bearing. The test rig is designed with 4 supports and 4 disks, two for each rotor. 


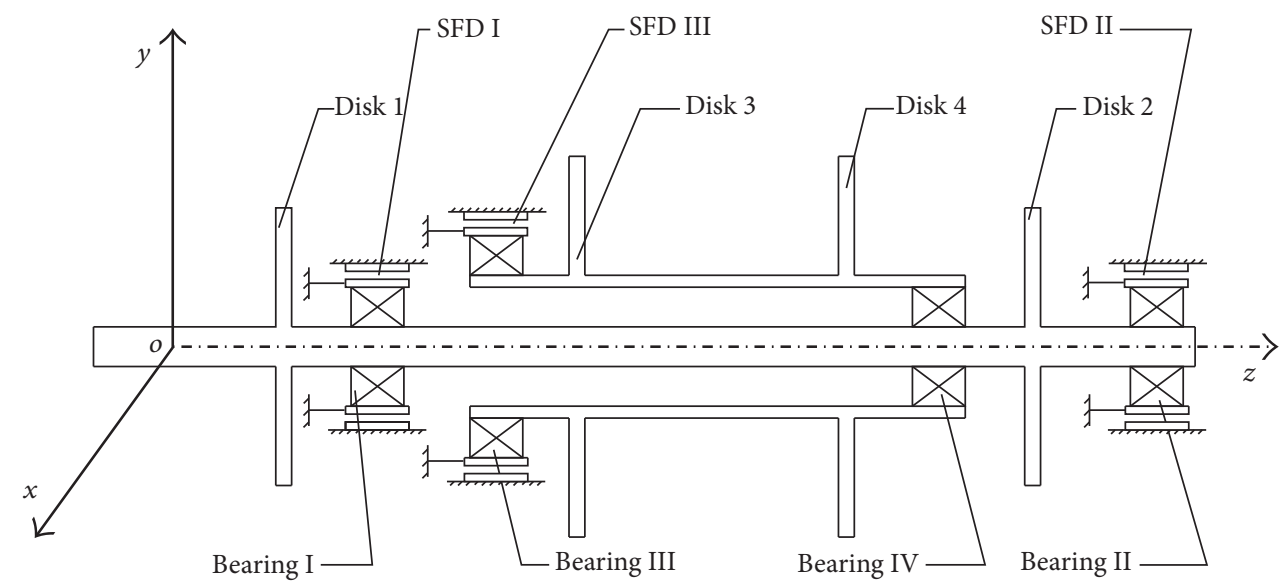

FIgURE 3: Structural diagram of the coaxial rotor system.

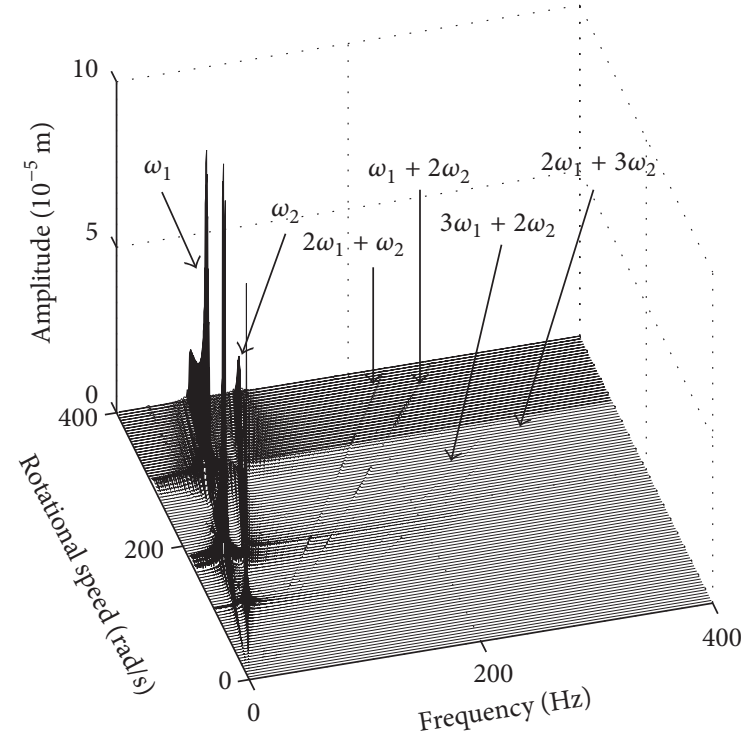

(a) Disk 2

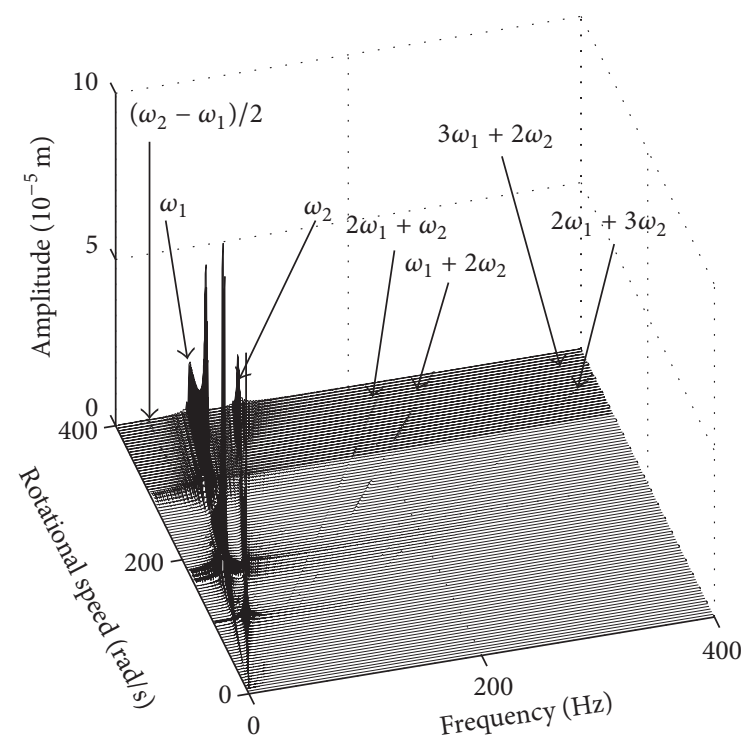

(b) Disk 4

FIGURE 4: Spectrum cascade of the horizontal response of disks 2 and 4, numerical simulation. $\lambda=-1.65$.

The rotors are supported by supports I, II, III, and IV. The squirrel cage + rolling bearing + SFD supporting scheme is adopted for supports I, II, and III. The intermediate bearing has one ring mounted on the shaft of inner rotor and the other ring on outer rotor as shown in Figure 3. The rotor comprises shaft and disks. Each shaft is driven by its own motor through flexible couplings and therefore their rotational speeds can be different.

Model parameters of the rotor system studied in this work are listed in Tables 1-5. Young's modulus of the shaft is $196 \mathrm{GPa}$, mass density is $7810 \mathrm{~kg} / \mathrm{m}^{3}$, and shear modulus is $75.5 \mathrm{GPa}$. To apply modal synthesis method, 40 modes are retained in the normal mode matrix $\phi_{\mathrm{k}}$. For the Newmarkbeta method, $\alpha=0.25$ and $\beta=0.5$.
Geometric dimensions and information of each element are listed in Table 1. Stiffness coefficients of elastic supports are listed in Table 2. Parameters of the intermediate bearing and SFDs are listed in Tables 3 and 4. The unbalance configuration and inertia properties of each disk are listed in Table 5.

\section{Numerical and Experimental Results}

Dynamic characteristics of both the co- and counterrotating dual-rotor system were studied in this section. The rotational speed ratio is defined as $\lambda=\omega_{2} / \omega_{1}$, where $\omega_{2}$ is the rotational speed of the outer rotor and $\omega_{1}$ is the rotational 
TABLE 1: Dimension and elements information of the rotor system.

\begin{tabular}{|c|c|c|c|c|c|}
\hline Node number & Axial location $(\mathrm{m})$ & Bearing/disk & Element number & Outer diameter $(\mathrm{m})$ & Inner diameter $(\mathrm{m})$ \\
\hline 1 & 0 & & 1 & 0.018 & 0.00 \\
\hline 2 & 0.08143 & & 2 & 0.018 & 0.00 \\
\hline 3 & 0.16286 & & 3 & 0.018 & 0.00 \\
\hline 4 & 0.24429 & & 4 & 0.018 & 0.00 \\
\hline 5 & 0.24909 & & 5 & 0.018 & 0.00 \\
\hline 6 & 0.25479 & & 6 & 0.018 & 0.00 \\
\hline 7 & 0.28879 & & 7 & 0.018 & 0.00 \\
\hline 8 & 0.32279 & & 8 & 0.018 & 0.00 \\
\hline 9 & 0.35879 & Disk number 1 & 9 & 0.018 & 0.00 \\
\hline 10 & 0.38369 & & 10 & 0.018 & 0.00 \\
\hline 11 & 0.40859 & & 11 & 0.018 & 0.00 \\
\hline 12 & 0.43349 & & 12 & 0.018 & 0.00 \\
\hline 13 & 0.43869 & Bearing number 1 & 13 & 0.018 & 0.00 \\
\hline 14 & 0.44479 & & 14 & 0.022 & 0.00 \\
\hline 15 & 0.54752 & & 15 & 0.022 & 0.00 \\
\hline 16 & 0.65025 & & 16 & 0.022 & 0.00 \\
\hline 17 & 0.75298 & & 17 & 0.022 & 0.00 \\
\hline 18 & 0.85571 & & 18 & 0.022 & 0.00 \\
\hline 19 & 0.95844 & & 19 & 0.022 & 0.00 \\
\hline 20 & 1.06117 & & 20 & 0.022 & 0.00 \\
\hline 21 & 1.06517 & Bearing number 4 & 21 & 0.022 & 0.00 \\
\hline 22 & 1.06867 & & 22 & 0.022 & 0.00 \\
\hline 23 & 1.08867 & & 23 & 0.022 & 0.00 \\
\hline 24 & 1.10867 & Disk number 2 & 24 & 0.022 & 0.00 \\
\hline 25 & 1.14274 & & 25 & 0.022 & 0.00 \\
\hline 26 & 1.17681 & & 26 & 0.022 & 0.00 \\
\hline 27 & 1.21088 & & 27 & 0.017 & 0.00 \\
\hline 28 & 1.21488 & Bearing number 2 & 28 & 0.014 & 0.00 \\
\hline 29 & 1.21838 & & 29 & 0.014 & 0.00 \\
\hline 30 & 1.23038 & \multicolumn{4}{|c|}{ End of inner rotor } \\
\hline 31 & 0.64200 & & 30 & 0.035 & 0.03 \\
\hline 32 & 0.66065 & & 31 & 0.035 & 0.03 \\
\hline 33 & 0.67930 & Bearing number 3 & 32 & 0.035 & 0.03 \\
\hline 34 & 0.68650 & & 33 & 0.038 & 0.03 \\
\hline 35 & 0.71170 & & 34 & 0.038 & 0.03 \\
\hline 36 & 0.73690 & & 35 & 0.038 & 0.03 \\
\hline 37 & 0.76210 & Disk number 3 & 36 & 0.038 & 0.03 \\
\hline 38 & 0.80784 & & 37 & 0.038 & 0.03 \\
\hline 39 & 0.85358 & & 38 & 0.038 & 0.03 \\
\hline 40 & 0.89932 & & 39 & 0.038 & 0.03 \\
\hline 41 & 0.94506 & & 40 & 0.038 & 0.03 \\
\hline 42 & 0.99080 & Disk number 4 & 41 & 0.038 & 0.03 \\
\hline 43 & 1.01430 & & 42 & 0.038 & 0.03 \\
\hline 44 & 1.02030 & & 43 & 0.070 & 0.03 \\
\hline 45 & 1.03330 & & 44 & 0.060 & 0.03 \\
\hline 46 & 1.06030 & & 45 & 0.060 & 0.03 \\
\hline 47 & 1.06430 & Bearing number 4 & 46 & 0.060 & 0.03 \\
\hline 48 & 1.07380 & & & & \\
\hline
\end{tabular}




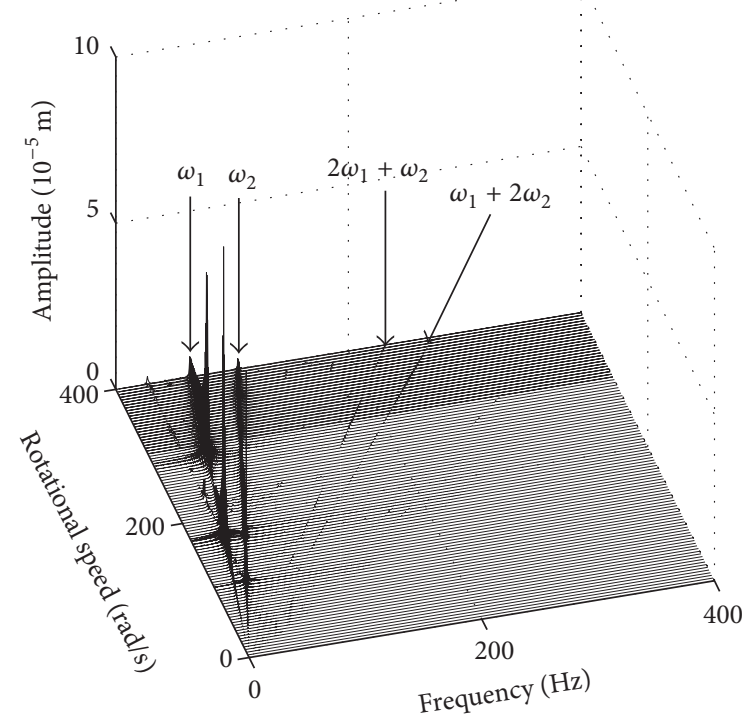

(a) Disk 2

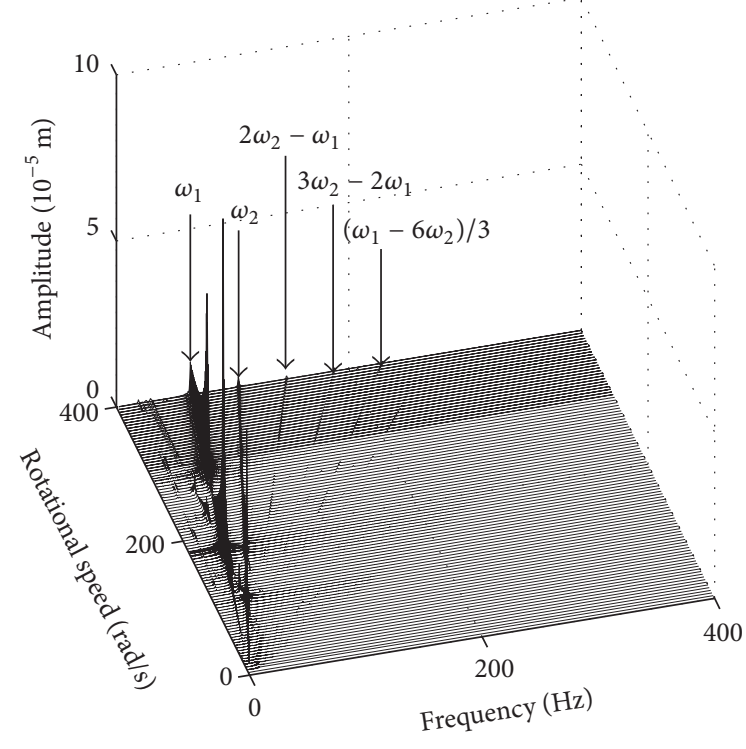

(b) Disk 4

FIGURE 5: Spectrum cascade of the horizontal response of disks 2 and 4 , numerical simulation. $\lambda=1.65$.

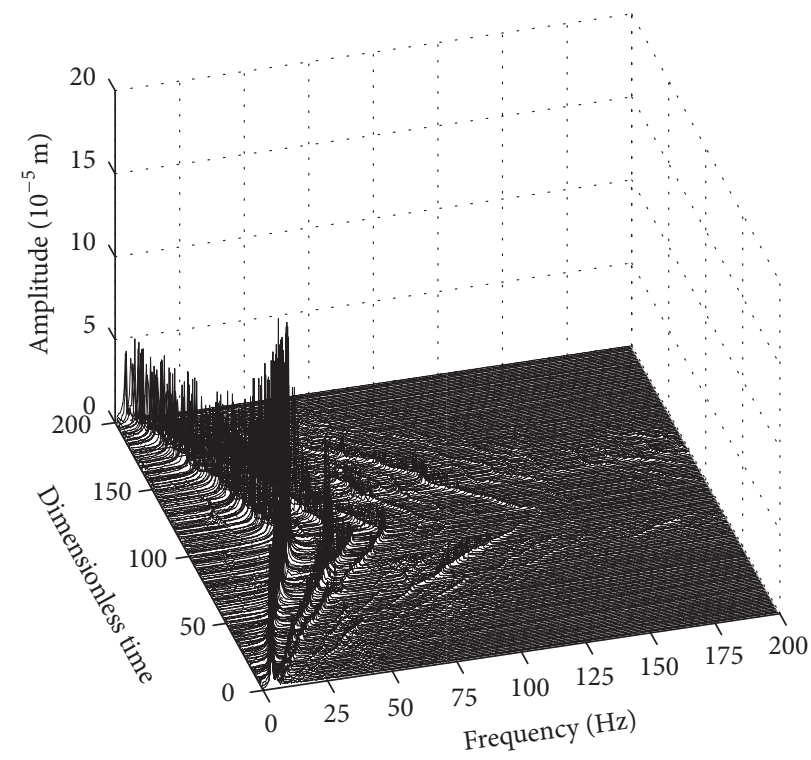

(a) Disk 2

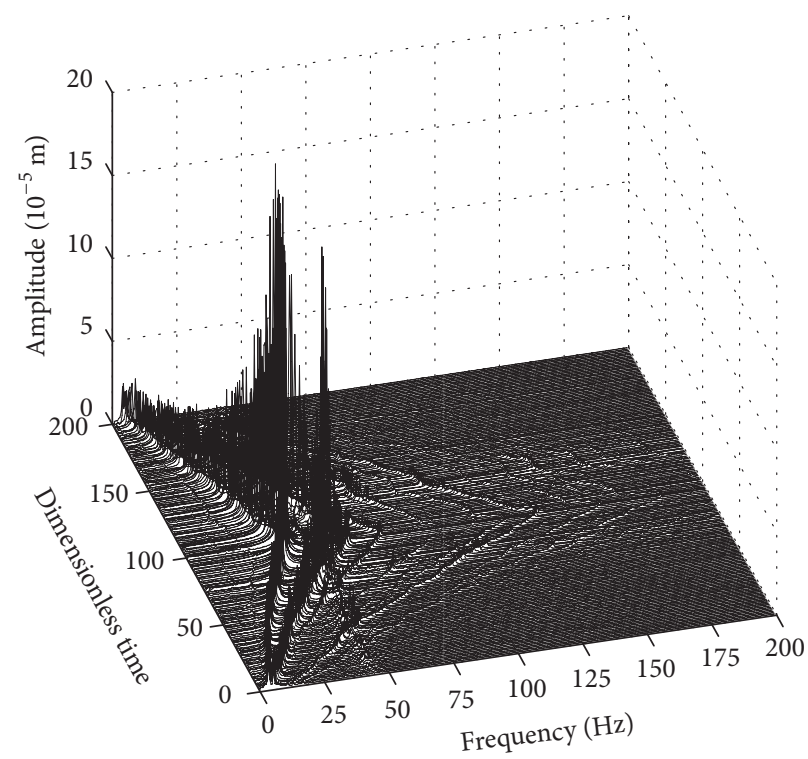

(b) Disk 4

FIGURE 6: Spectrum cascade of the horizontal response of disks 2 and 4, experimental results. $\lambda=-1.65$.

TABLE 2: Stiffness of elastic supports (squirrel cage).

Support I $\quad$ Support II Support III Support IV

Stiffness (N/m) $1.45 \times 10^{6} \quad 2.21 \times 10^{5} \quad 9.29 \times 10^{5} \quad-$

speed of the inner rotor. $\lambda=1.65$ (corotating) and $\lambda=-1.65$ (counterrotating) cases are studied in the current work.
4.1. Overview of the Results. With the model established by method described in Section 2, steady-state unbalance response of the rotor system is obtained for rotational speed of the inner rotor varying from $4 \mathrm{rad} / \mathrm{s}$ to $800 \mathrm{rad} / \mathrm{s}$ with a step length of $2 \mathrm{rad} / \mathrm{s}$. Zero initial condition is adopted for the first step. For the rest of the steps, result of previous step is adopted as the initial condition. The critical speeds of the rotor system can be identified by plotting and analyzing the 


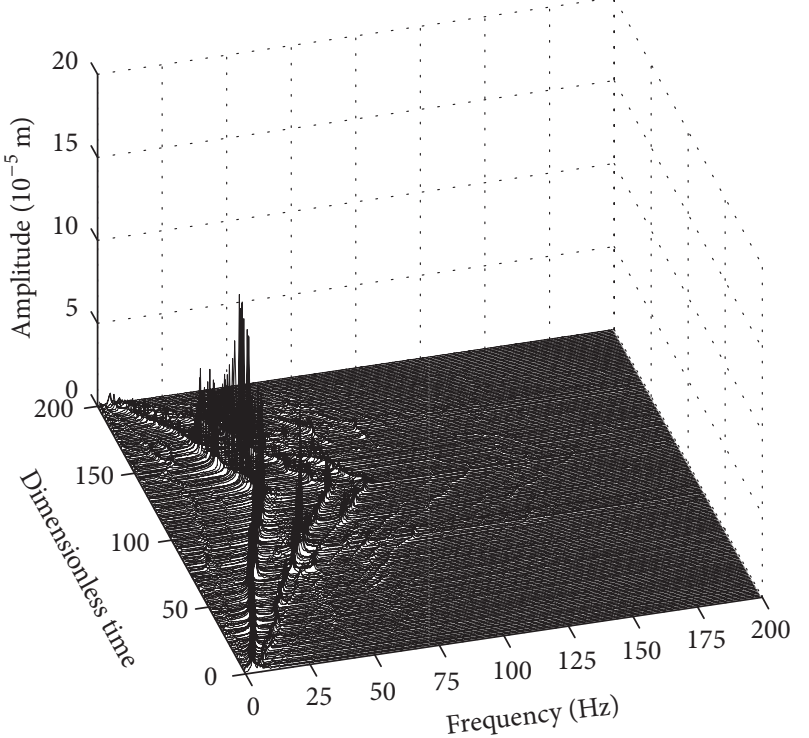

(a) Disk 2

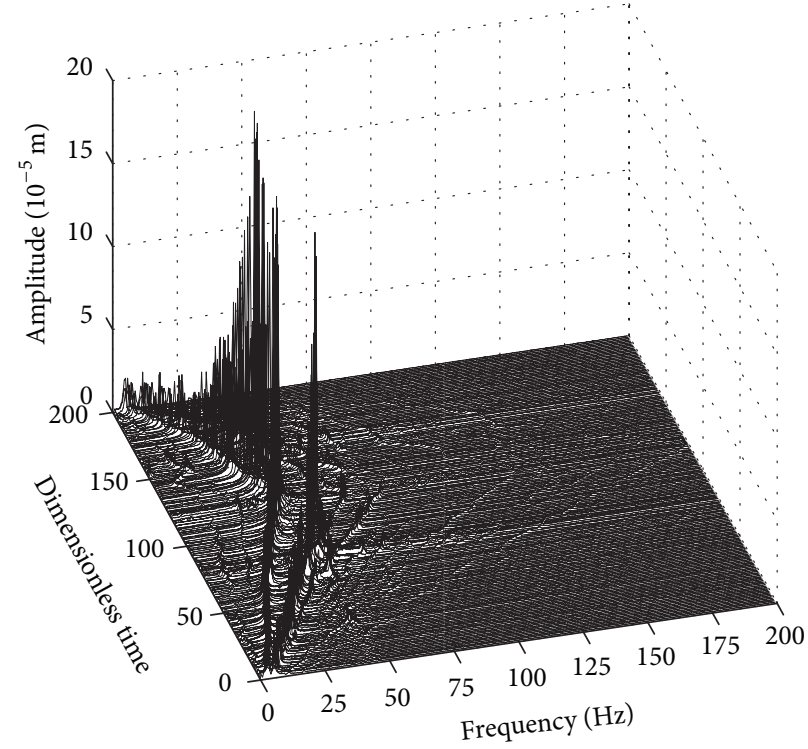

(b) Disk 4

FIGURE 7: Spectrum cascade of the horizontal response of disks 2 and 4, experimental results. $\lambda=1.65$.

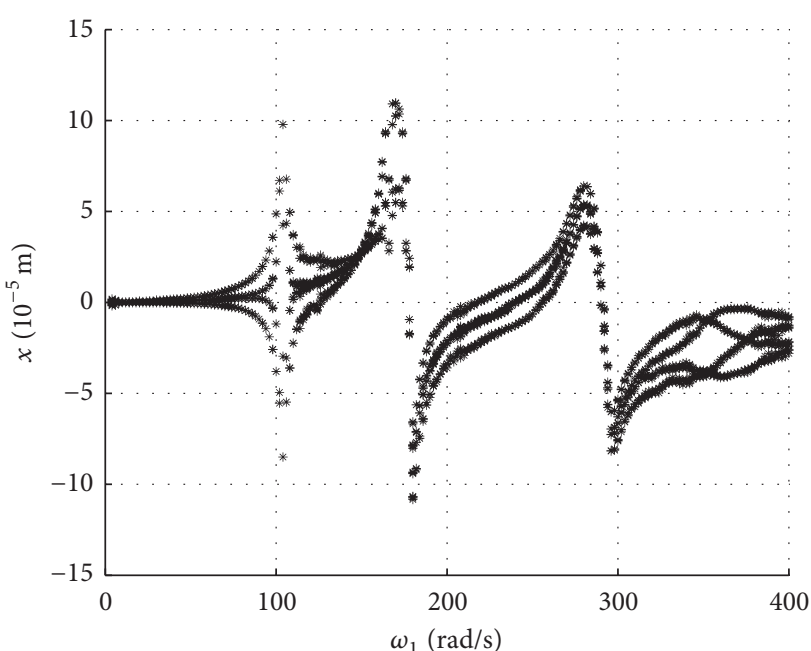

(a) Disk 2

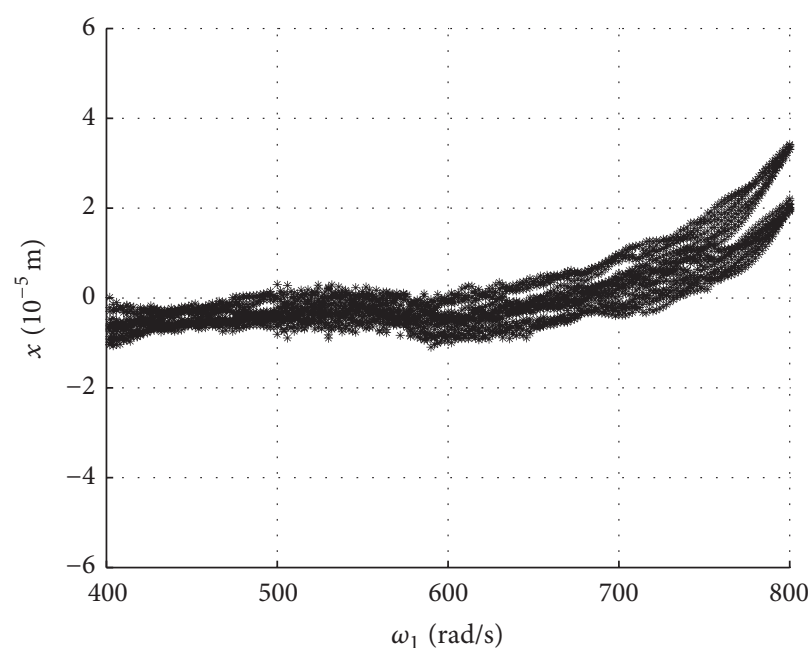

(c) Disk 2

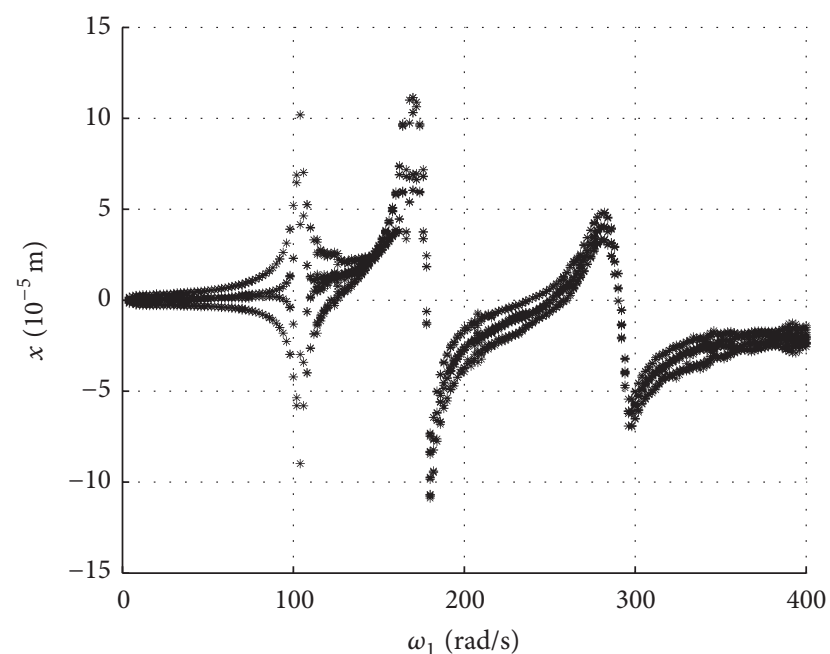

(b) Disk 4

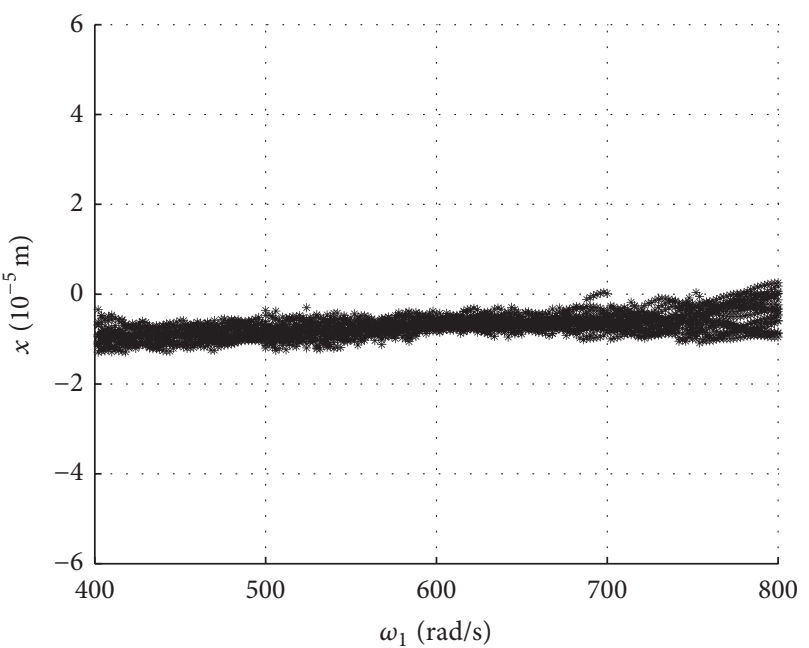

(d) Disk 4

FIGURE 8: Bifurcation diagrams of the horizontal response of disk 2 and disk 4 with rotor speed. $\lambda=-1.65$. 
TABle 3: Parameters of the intermediate bearing.

\begin{tabular}{lcccc}
\hline Radius of inner ring $(\mathrm{mm})$ & Radius of outer ring $(\mathrm{mm})$ & Number of rollers & Contact stiffness $\left(\mathrm{N} / \mathrm{m}^{3 / 2}\right)$ & Radial clearance $(\mu \mathrm{m})$ \\
\hline 9.37 & 14.13 & 9 & $7.055 \times 109$
\end{tabular}

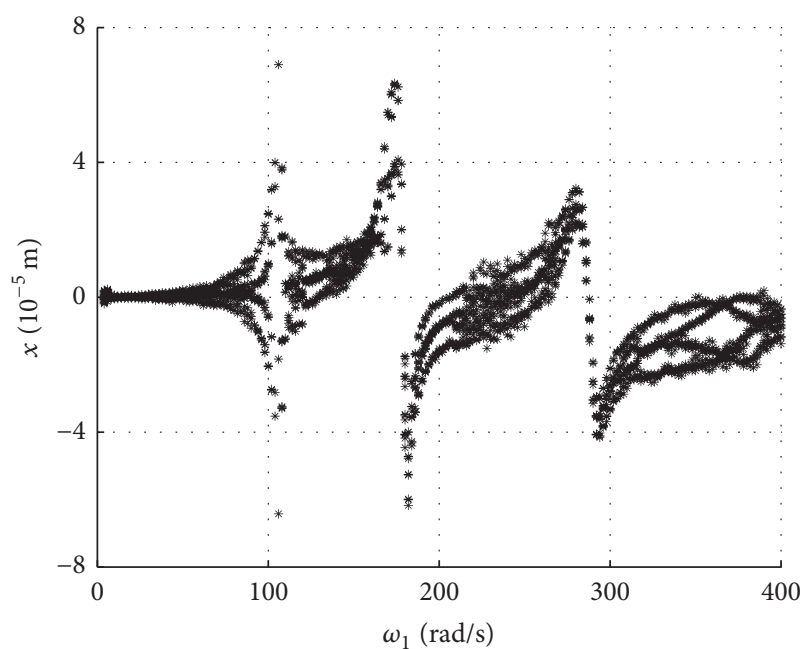

(a) Disk 2

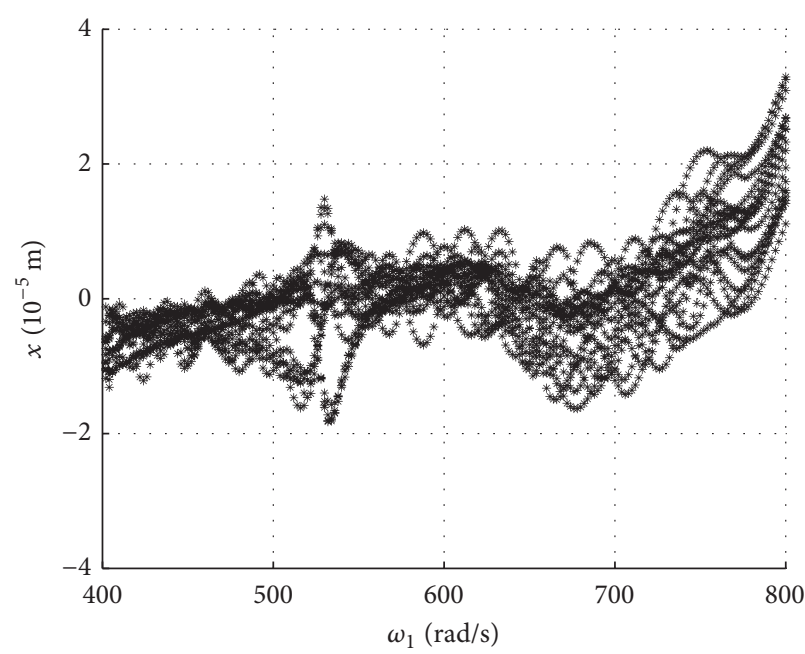

(c) Disk 2

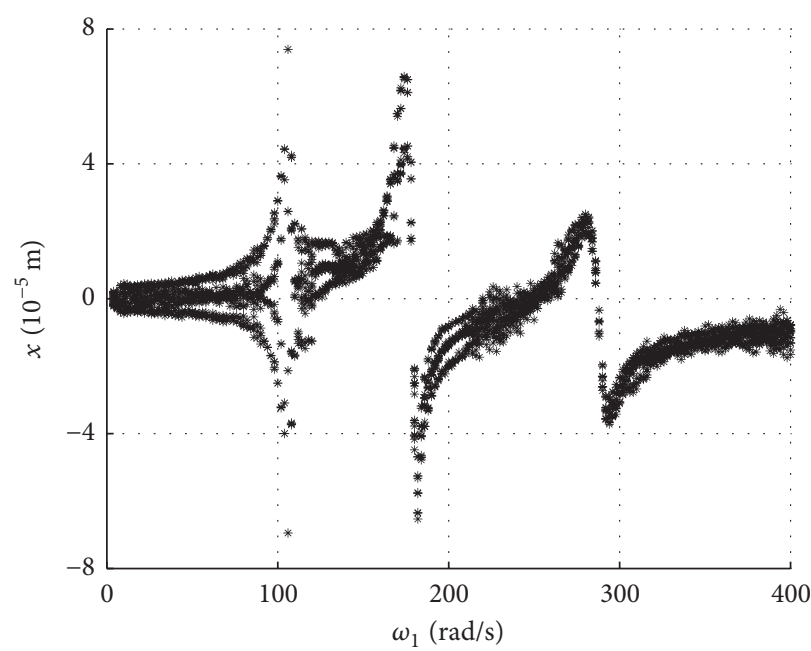

(b) Disk 4

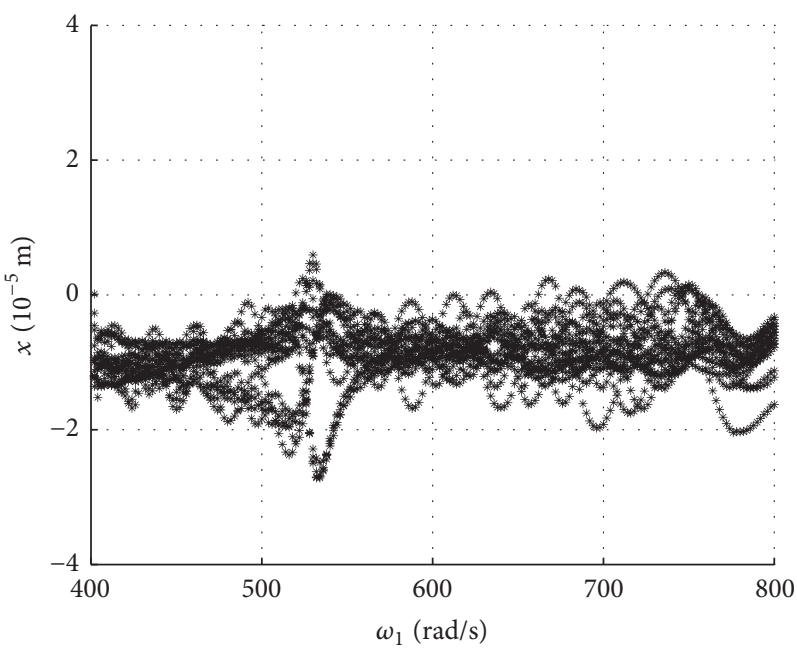

(d) Disk 4

FIGURE 9: Bifurcation diagrams of the horizontal response of disk 2 and disk 4 with rotor speed. $\lambda=1.65$.

TABLE 4: Parameters of SFDs.

\begin{tabular}{lccc}
\hline & \multicolumn{2}{c}{ Inner rotor } & Outer rotor \\
& SFD I & SFD II & SFD III \\
\hline Radius $\mathrm{R} / \mathrm{mm}$ & 25 & 18 & 35 \\
Length $\mathrm{L} / \mathrm{mm}$ & 15 & 15 & 20 \\
Radial clearance c/mm & 0.1 & 0.1 & 0.08 \\
Dynamic viscosity $\mu_{s} / 10^{-2} \mathrm{~Pa} \cdot \mathrm{s}$ & \multicolumn{4}{c}{1.0752} \\
\hline
\end{tabular}

TABLE 5: Unbalance configuration and inertia properties of disks.

\begin{tabular}{lcccc}
\hline & \multicolumn{2}{c}{ Inner rotor } & \multicolumn{2}{c}{ Outer rotor } \\
& Disk 1 & Disk 2 & Disk 3 & Disk 4 \\
\hline Unbalance $\left(\times 10^{-5} \mathrm{~kg} \cdot \mathrm{m}\right)$ & 2 & 4 & 1 & 2 \\
$\begin{array}{l}\text { Mass }(\mathrm{kg}) \\
\text { Polar moment of inertia } \\
\left(\mathrm{kg} \cdot \mathrm{m}^{2}\right)\end{array}$ & 2.3386 & 2.3386 & 3.2590 & 1.6303 \\
\hline
\end{tabular}




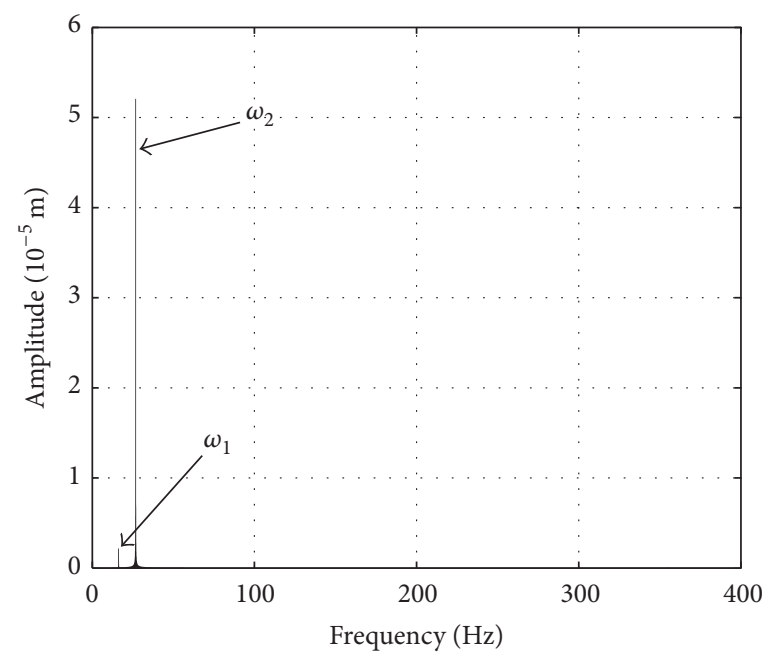

(a) Spectrogram

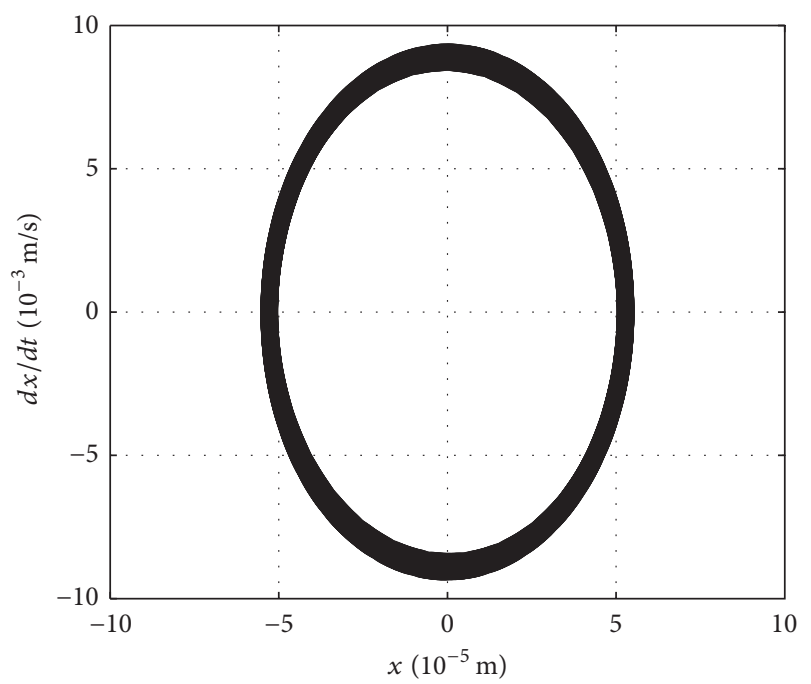

(c) Phase trajectory

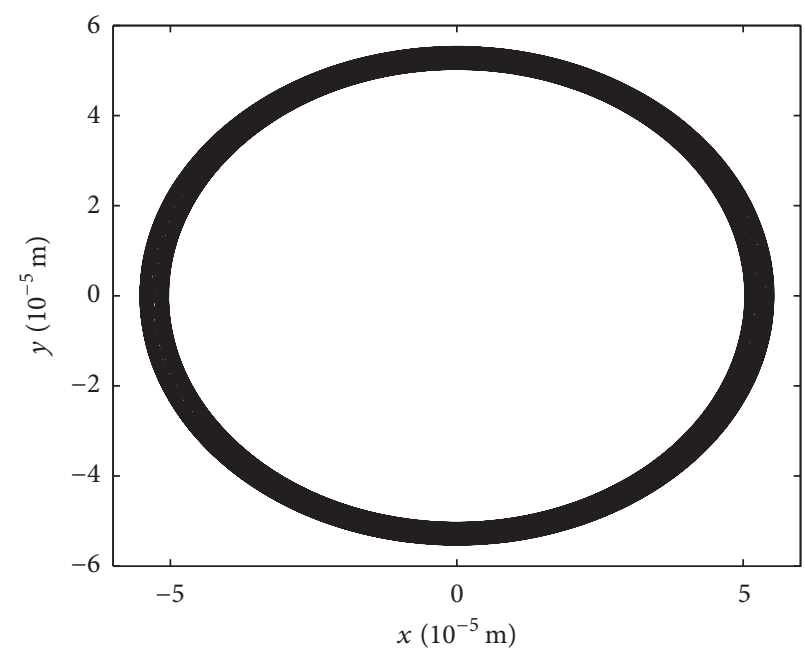

(b) Orbit

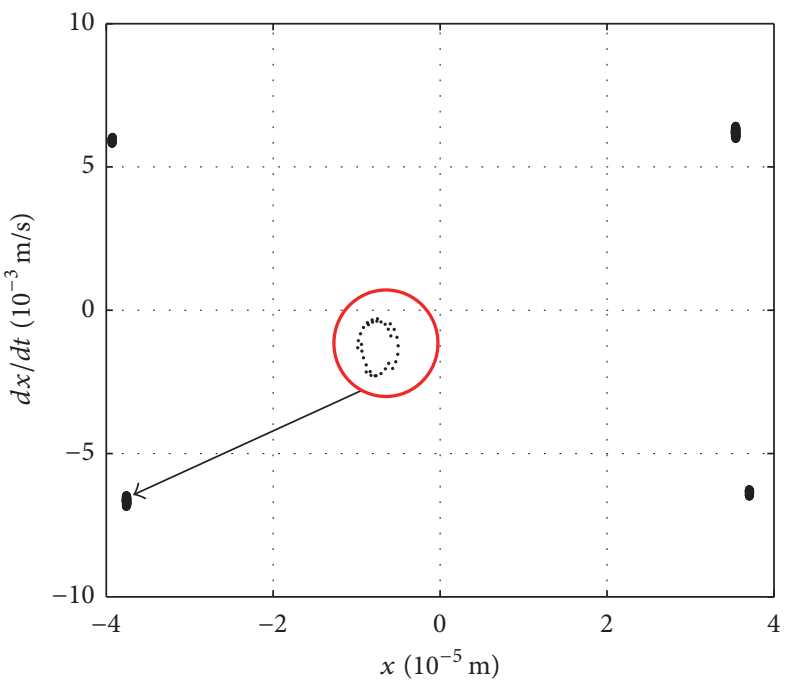

(d) Poincare map

FIGURE 10: Response analysis of disk 2: $\omega_{1}=102 \mathrm{rad} / \mathrm{s}$ and $\lambda=-1.65$.

spectrum cascade of the numerical and experimental results. Without loss of generality, only unbalance response of disk 2 and disk 4 are analyzed.

Numerical results of the spectrum cascades for the horizontal response of disk 2 and disk 4 with inner rotor operating in 4-400 rad/s are shown in Figures 4 and 5. And the corresponding experimental results are shown in Figures 6 and 7. The following conclusions can be reached from Figures 4-7:

(1) Responses of the inner and outer rotor are coupled because of the intermediate bearing. Frequency components, $\omega_{1}$ and $\omega_{2}$, corresponding to the unbalance excitation frequencies of the inner and outer rotor, are dominant in the response of disk 2 and disk 4. The coupling effect which makes the spectrum cascades corresponding to disk 2 and disk 4 basically follows the same pattern with only slight differences in amplitude.

(2) Frequency components besides $\omega_{1}$ and $\omega_{2}$ in the responses of disks 2 and 4 , such as $2 \omega_{1}+\omega_{2}, \omega_{1}+2 \omega_{2}$, $3 \omega_{1}+2 \omega_{2}, 2 \omega_{1}+3 \omega_{2}$, and $\left(\omega_{2}-\omega_{1}\right) / 2$, are mainly caused by the nonlinear forces of the SFD and the coupling effect of the intermediate bearing. However, the combination frequency components are different for co- and counterrotating systems. For example, Figures 4 and 5 show the spectrum cascade of disks 2 and 4 under counter- and corotating conditions. In Figure $4(\mathrm{a})$, the combination frequency components are $2 \omega_{1}+\omega_{2}, \omega_{1}+2 \omega_{2}, 3 \omega_{1}+2 \omega_{2}$, and $2 \omega_{1}+3 \omega_{2}$, while in Figure 5(a) only $2 \omega_{1}+\omega_{2}$ and $\omega_{1}+2 \omega_{2}$ are observed. In Figure $4(\mathrm{~b})$, the combination frequency components are $2 \omega_{1}+\omega_{2}, \omega_{1}+2 \omega_{2}, 3 \omega_{1}+2 \omega_{2}, 2 \omega_{1}+3 \omega_{2}$, and 


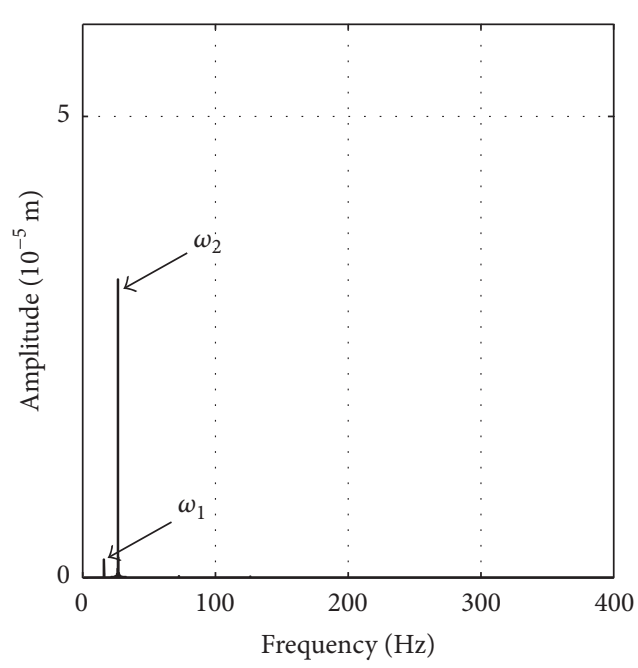

(a) Spectrogram

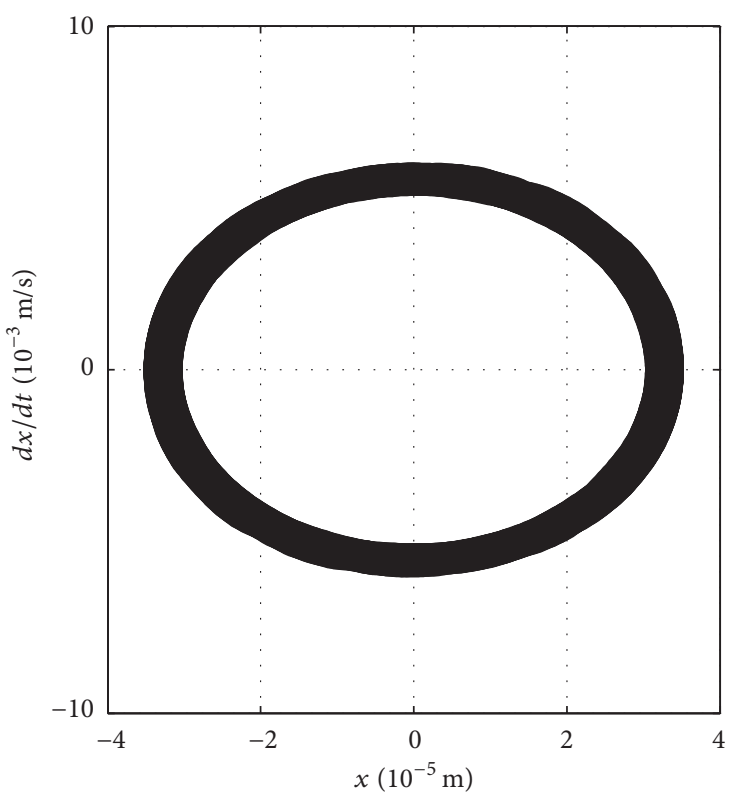

(c) Phase trajectory

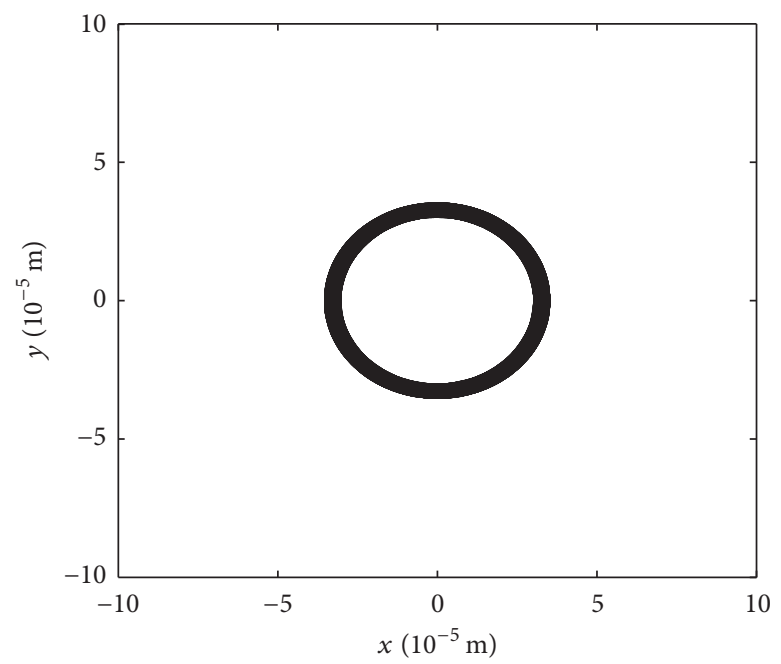

(b) Orbit

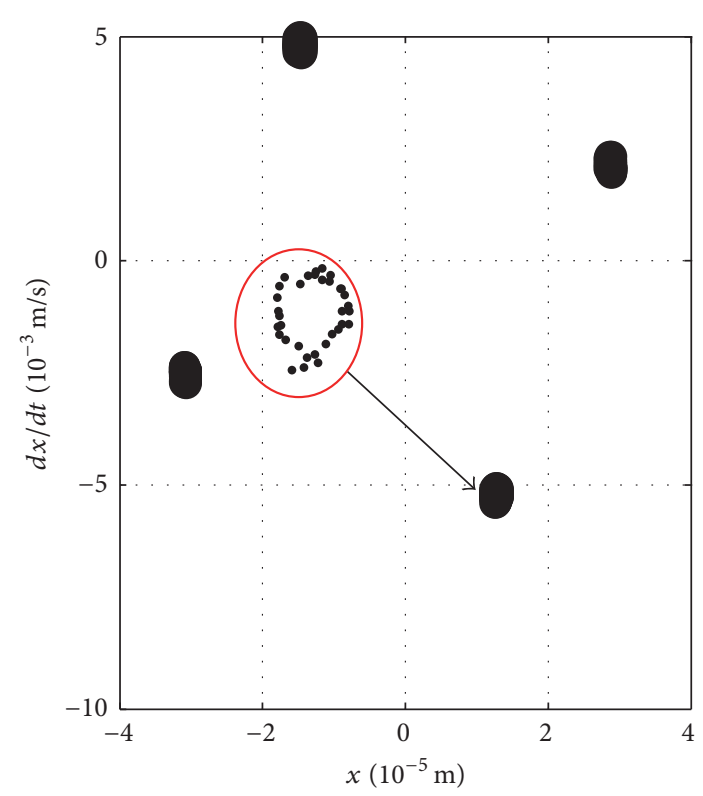

(d) Poincare map

FIGURE 11: Response analysis of disk 2: $\omega_{1}=102 \mathrm{rad} / \mathrm{s}$ and $\lambda=1.65$.

$\left(\omega_{2}-\omega_{1}\right) / 2$ while in Figure 5(b) those combinations are $2 \omega_{2}-\omega_{1}, 3 \omega_{2}-2 \omega_{1}$, and $\left(6 \omega_{2}+\omega_{1}\right) / 3$.

(3) In Figure 4, two critical speeds of the counterrotating rotor system, $168 \mathrm{rad} / \mathrm{s}$ and $186 \mathrm{rad} / \mathrm{s}$, are observed. For the corotating system, Figure 5, the corresponding critical speeds are $168 \mathrm{rad} / \mathrm{s}$ and $190 \mathrm{rad} / \mathrm{s}$. From the experimental results presented in Figures 6 and 7, the lowest two critical speeds for counter- and corotating systems are $170 \mathrm{rad} / \mathrm{s}$ and $189 \mathrm{rad} / \mathrm{s}$ and $173 \mathrm{rad} / \mathrm{s}$ and $195 \mathrm{rad} / \mathrm{s}$. The differences of critical speeds for co- and counterrotating systems are mainly caused by the gyroscopic effect. For the corotating rotor system, the gyroscopic moments of both rotors act in the same direction to strengthen the shaft thus raising the critical speeds. While, for the counterrotating system, the gyroscopic moments act in the opposite directions; thus the strengthening effect is weakened and then lower critical speeds are achieved.

4.2. Unbalance Response Analysis. Respectively, for counterand corotating cases, Figures 8 and 9 show bifurcation diagrams for horizontal displacement of disk 2 and disk 4 with rotor speed. Sampling period for the bifurcation diagram is $5 \times 2 \pi / \omega_{1}$ because the rotational speed ratio of outer and inner rotor is \pm 1.65 and $5 \approx 1.65 \times 3$. The horizontal axes are rotational speeds of inner and outer rotor. And the vertical axes are horizontal displacement of disk 2 and disk 4 . 


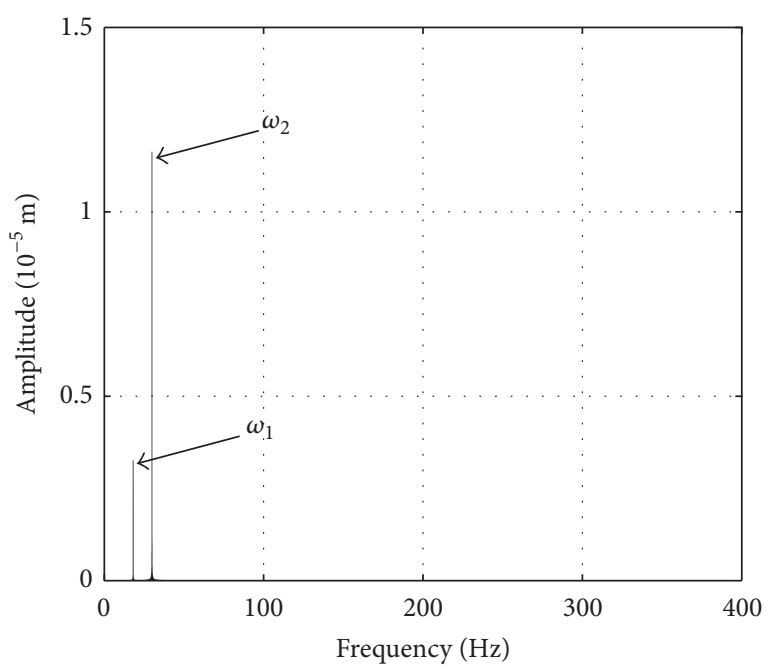

(a) Spectrogram

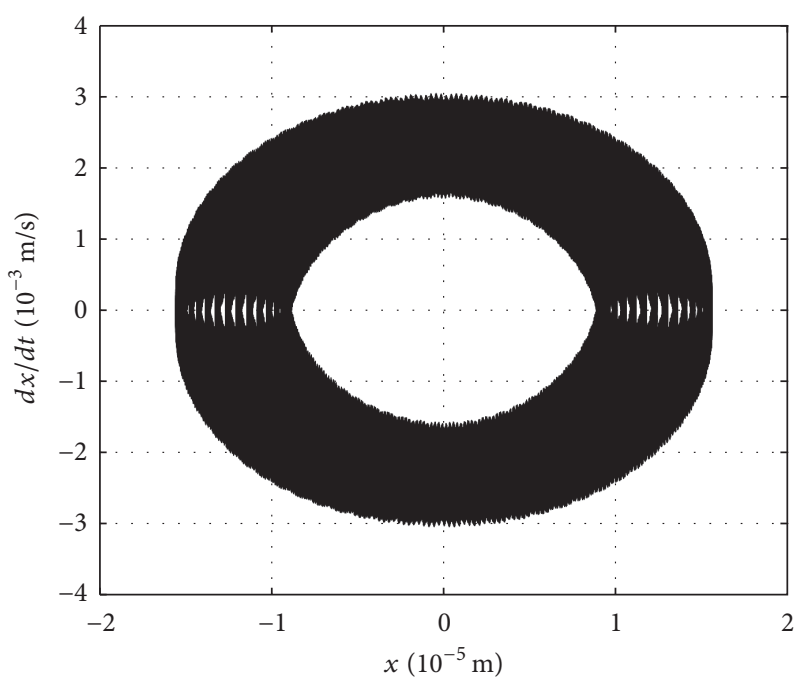

(c) Phase trajectory

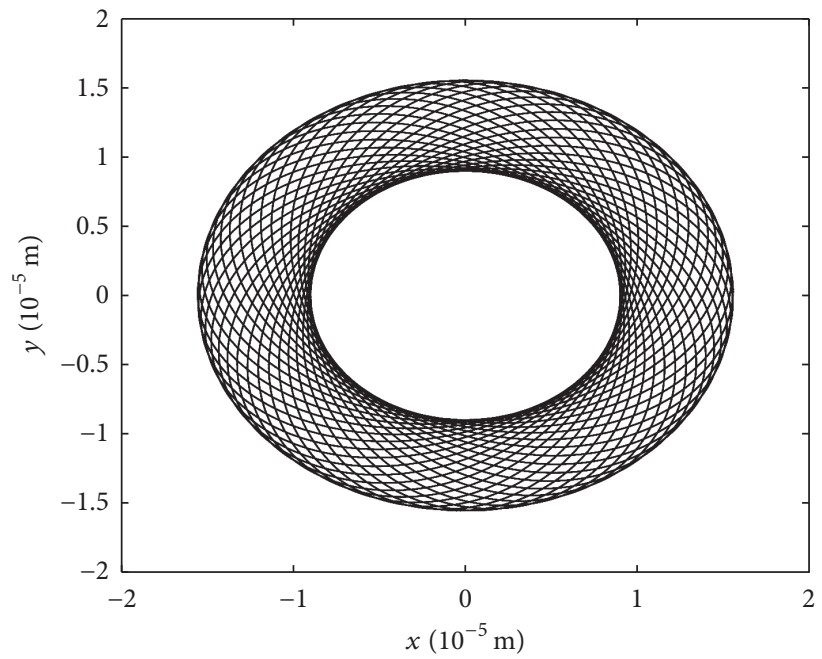

(b) Orbit

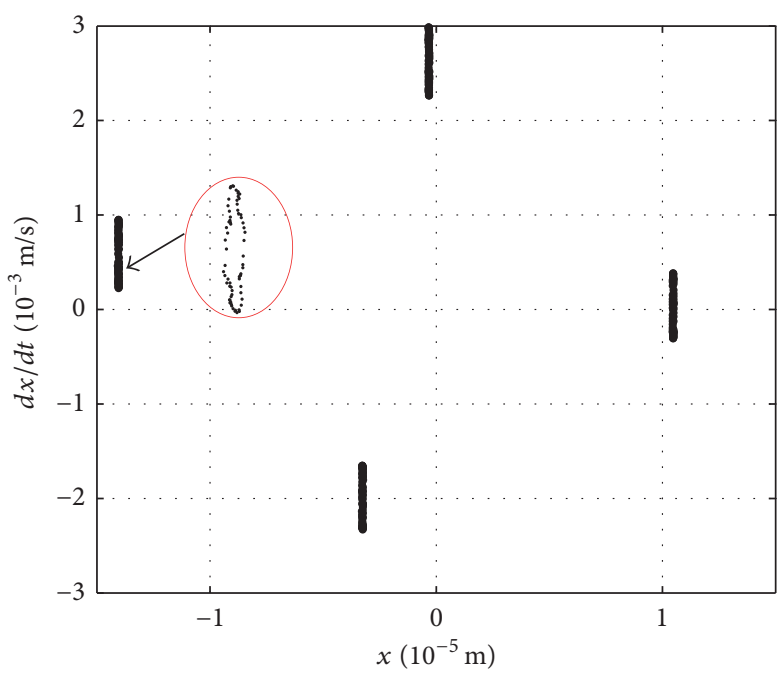

(d) Poincare map

FIGURE 12: Response analysis of disk 2: $\omega_{1}=114 \mathrm{rad} / \mathrm{s}$ and $\lambda=-1.65$.

Basically, disks 2 and 4 execute multiple period orbital motion around critical speeds, which can be seen from the bifurcation diagrams shown in Figures 8 and 9. For both the counter- and corotating cases, when the rotor systems operate near the critical speeds, a four-period orbital motion is observed; see Figures 10, 11, 16, and 17. Although only four scattered and isolated points are observed in the Poincare maps shown in Figures 10(d), 11(d), 16(d), and 17(d), the enlarged graphic circulated by the red circle shows that the isolated "points" are actually closed loops which indicate quasi-periodic motion. Besides, by comparison between Figures 10 and 11 it can be seen that both the counter- and corotating systems execute four-period orbital motion although their orbits show a little difference-the amplitude for the orbit of disk 2 of the counterrotating system is slightly larger than that of the corotating system. Also, only $\omega_{1}$ and $\omega_{2}$ are observed in the spectrogram shown in Figures 10(a) and 11(a).
And the amplitude of $\omega_{2}$ is much larger than that of $\omega_{1}$, which means the outer rotor is the main source of vibration.

As the rotational speed rises to $114 \mathrm{rad} / \mathrm{s}$, notable differences for the spectrogram, orbit, phase trajectory, and Poincare map between the counter- and corotating conditions are observed, Figures 12 and 13. The Poincare map shown in Figure 12(d) suggests that disk 2 executes fourperiod quasi-periodic motion under counterrotating condition while no obvious periodicity can be observed from the random-like and irregularly distributed points shown in Figure 13(d), the corotating condition. In contrast, this phenomenon is reversed when the rotational speed is further increased to $140 \mathrm{rad} / \mathrm{s}$; see Figures 14 and 15 . Under $140 \mathrm{rad} / \mathrm{s}$, the corotating case, Figure 15(d), indicates a fourperiod quasi-periodic motion while the counterrotating case, Figure $14(\mathrm{~d})$, shows randomly points cloud. Besides, from 


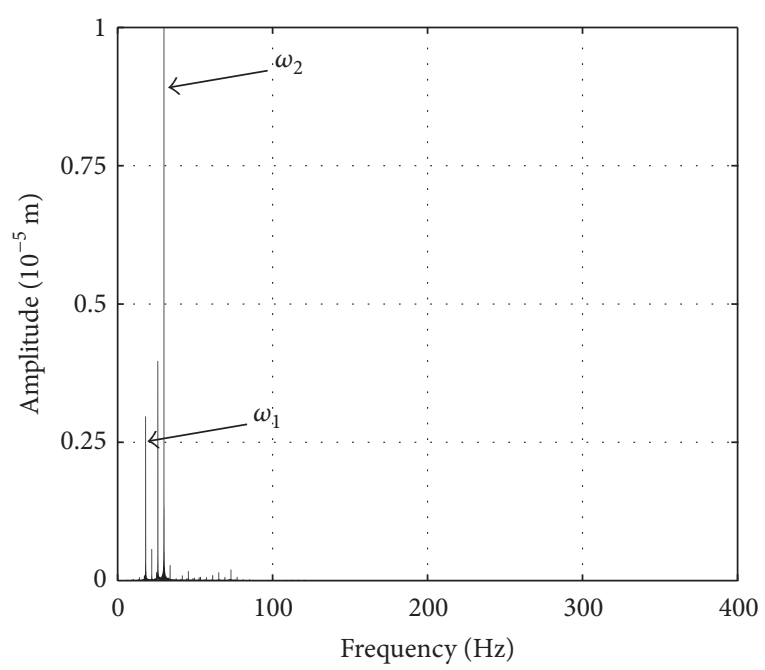

(a) Spectrogram

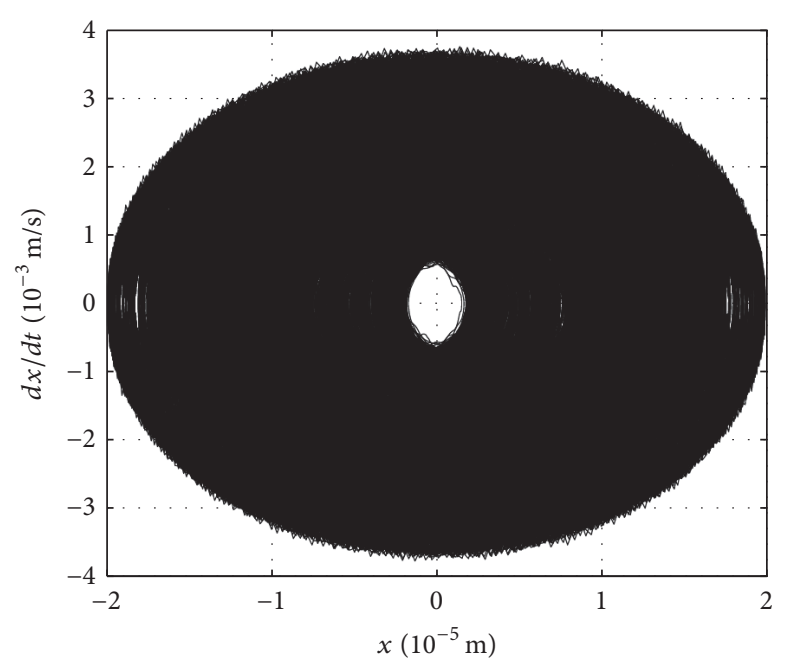

(c) Phase trajectory

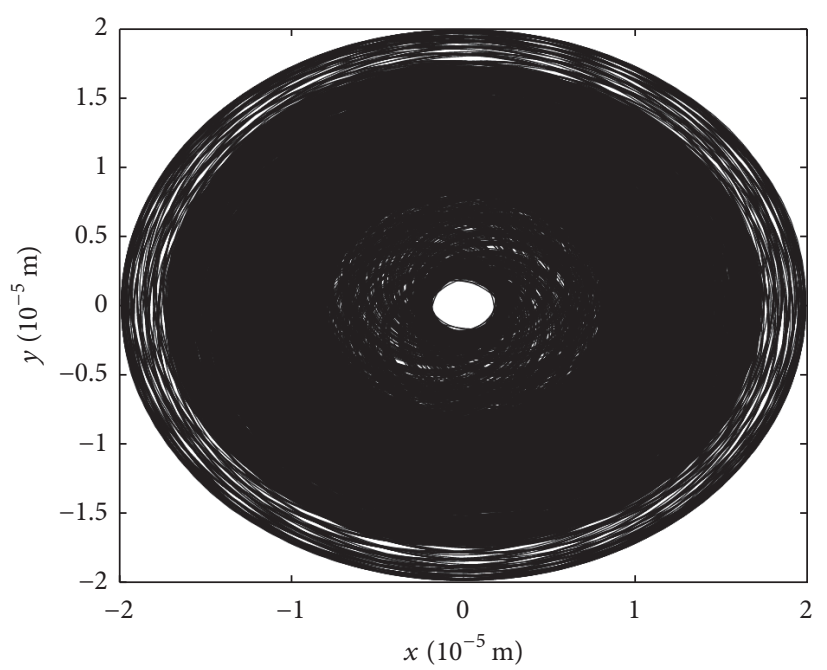

(b) Orbit

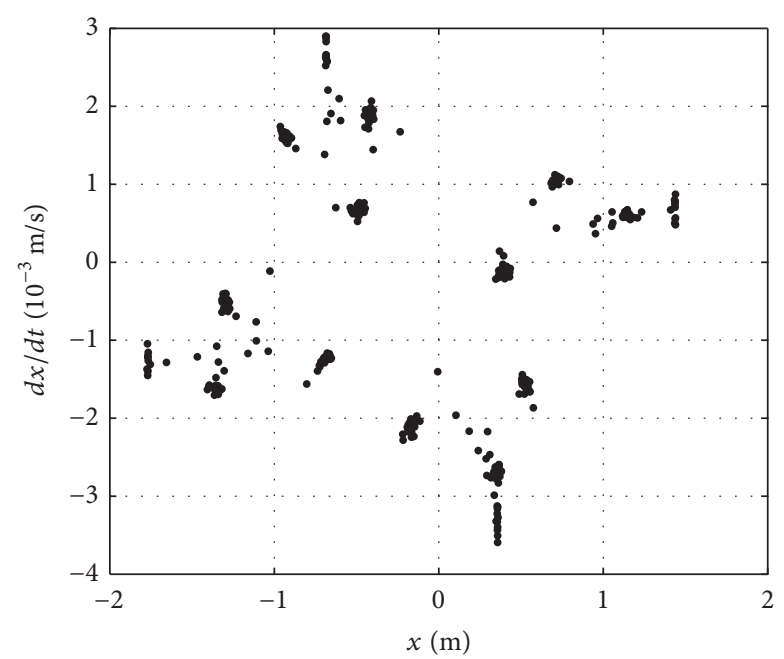

(d) Poincare map

FIGURE 13: Response analysis of disk 2: $\omega_{1}=114 \mathrm{rad} / \mathrm{s}$ and $\lambda=1.65$.

Figures 13(a) and 15(a) it can be seen that frequency components other than $\omega_{1}$ and $\omega_{2}$ can be found for the conditions when no obvious periodicity can be observed.

At $170 \mathrm{rad} / \mathrm{s}$, approximately the next critical speed, fourperiod quasi-periodic motion emerges again; see Figures 16 and 17. Also, it is noted that the closed loop shown in Figure 16(d), the counterrotating case, is twisted while that of the corotating case, Figure $17(\mathrm{~d})$, is an ellipse. Figures 16 and 17 also reveal that the amplitude of disk 2 under counterrotating condition is approximately twice that for the corotating condition.

To conclude, the rotors execute four-period quasiperiodic motion around critical speeds, Figures 10, 11, 16, and 17. Between two critical speeds, the motion state of the rotor system can be quite different for the counter- and corotating cases, Figures 12-15. Around critical speeds, the amplitude of disk 2 is much larger for the counterrotating case comparing with that of the corotating case, Figures 10(b), 11(b), 16(b), and 17(b).

4.3. Experimental Validation. The comparison between numerical and experimental results has already been made in the previous section, Figures 4-7. Orbits of disk 2 and 4 under three different rotational speeds are compared in this section for further validation; see Figures 18-22. The numerical results show great agreement with the experimental results, which validate the model and method adopted in the current work.

\section{Conclusions}

A modeling method combining the finite element method and the fixed interface modal synthesis method has been developed in the work to establish the nonlinear model of 


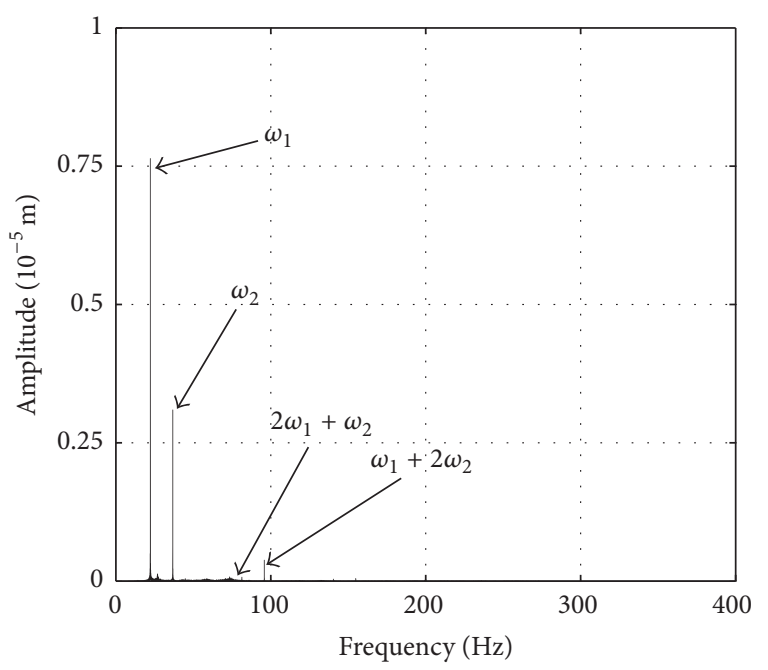

(a) Spectrogram

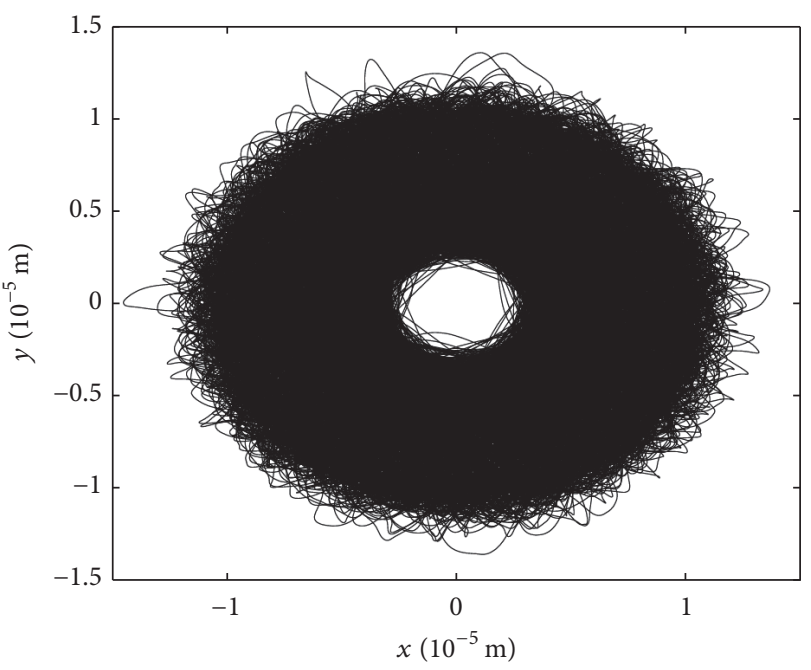

(b) Orbit

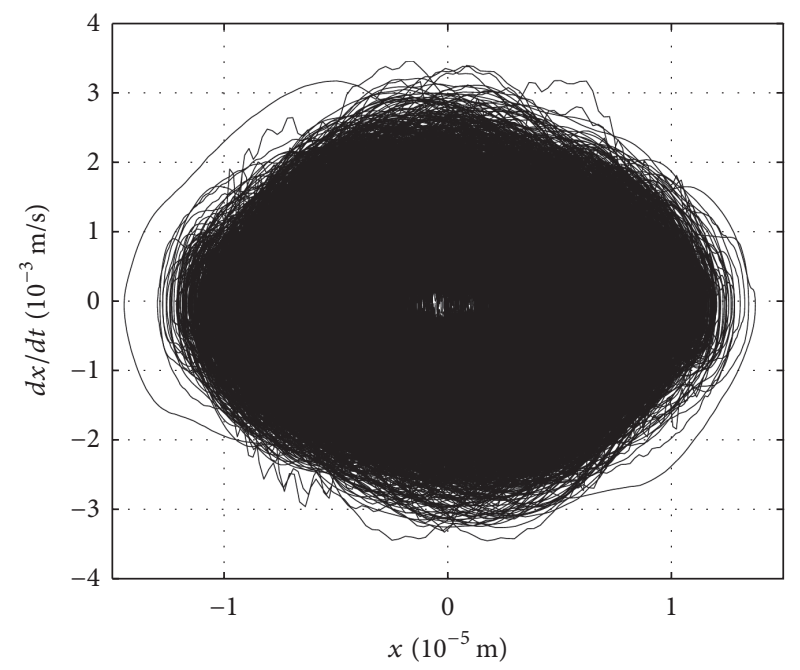

(c) Phase trajectory

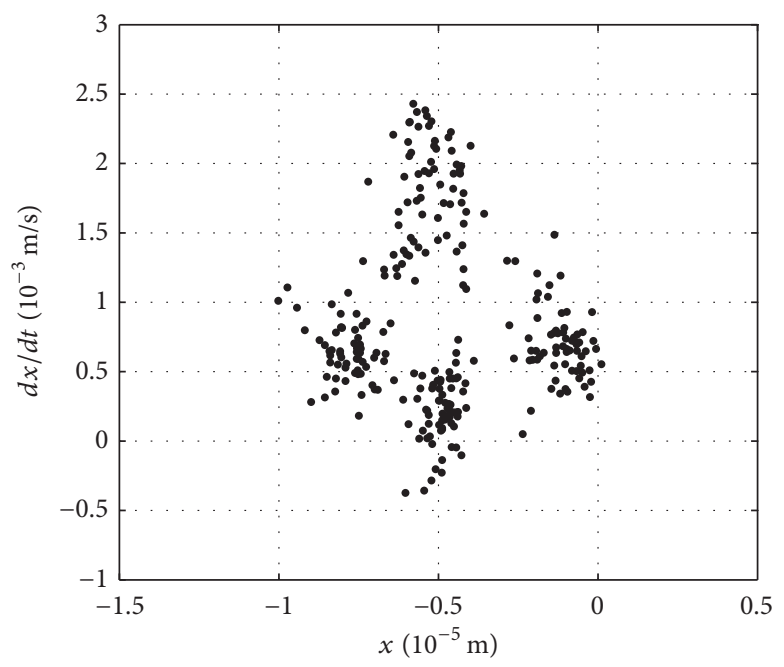

(d) Poincare map

FIGURE 14: Response analysis of disk 2: $\omega_{1}=140 \mathrm{rad} / \mathrm{s}$ and $\lambda=-1.65$. 


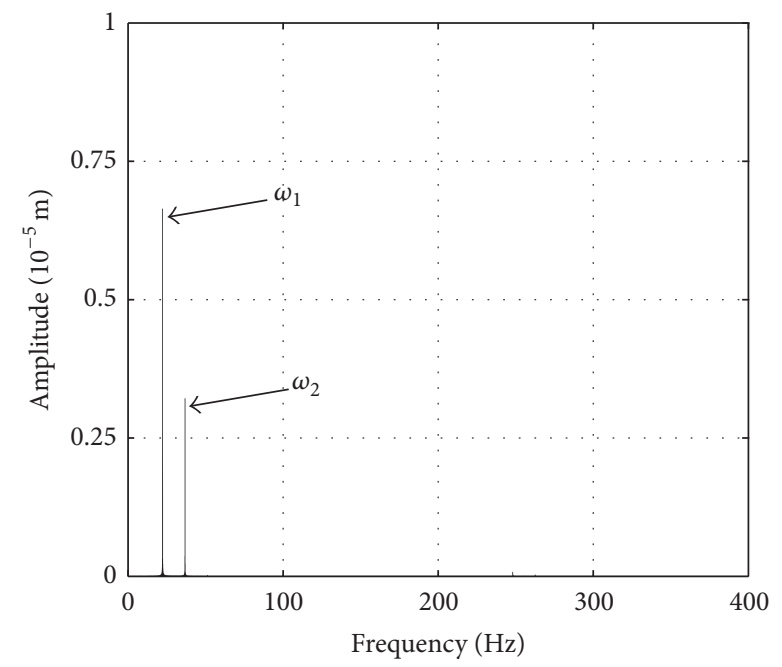

(a) Spectrogram

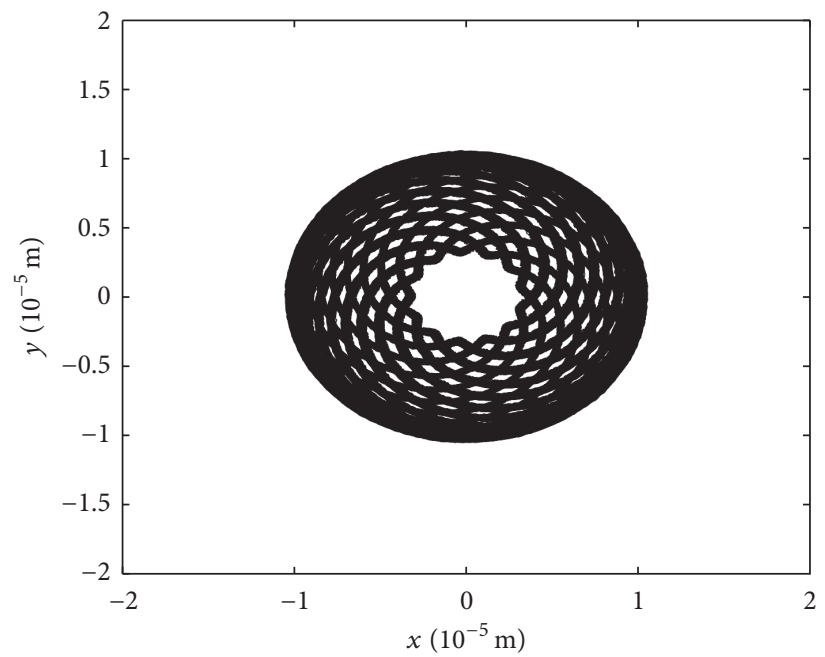

(b) Orbit

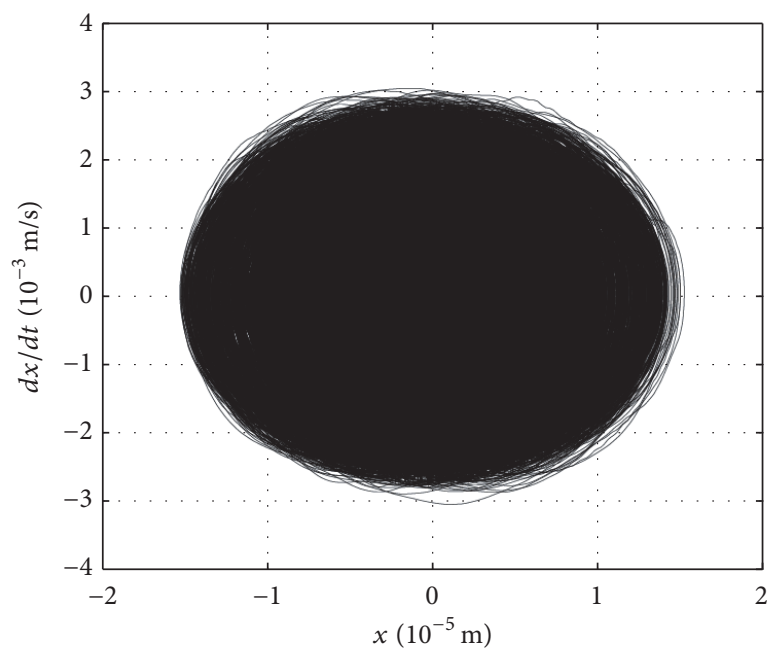

(c) Phase trajectory

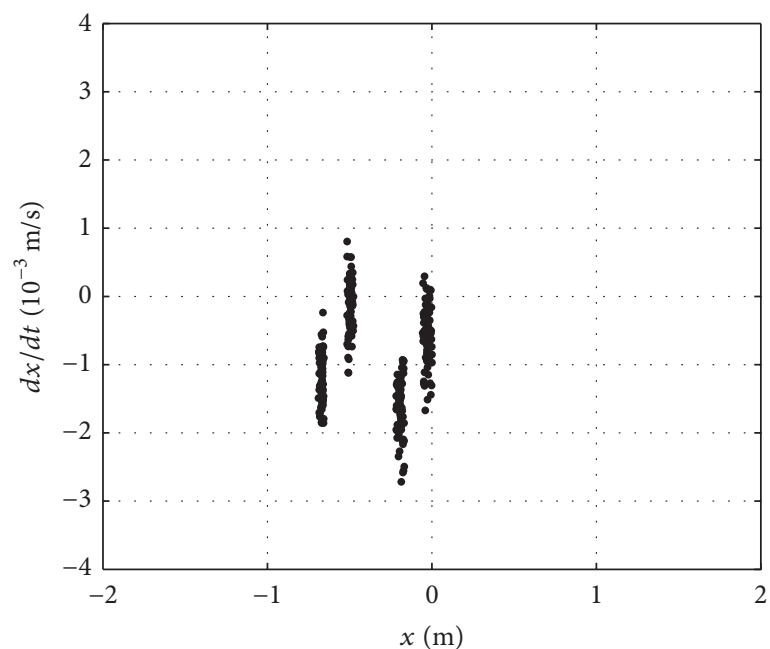

(d) Poincare map

FIGURE 15: Response analysis of disk 2: $\omega_{1}=140 \mathrm{rad} / \mathrm{s}$ and $\lambda=1.65$. 


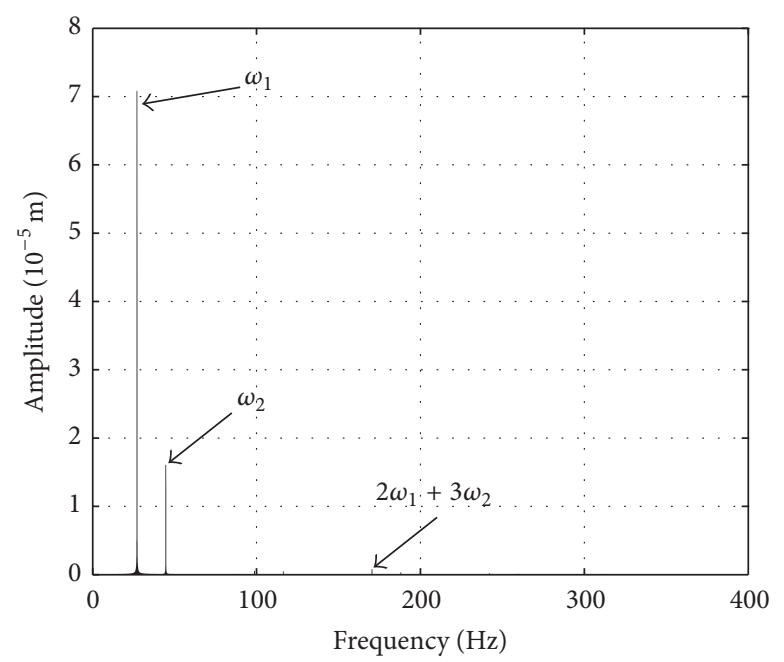

(a) Spectrogram

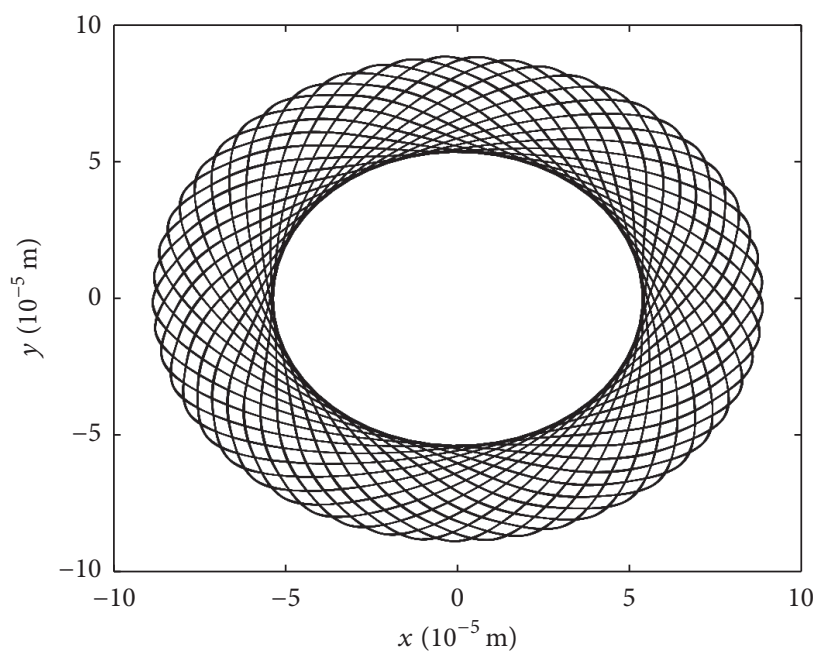

(b) Orbit

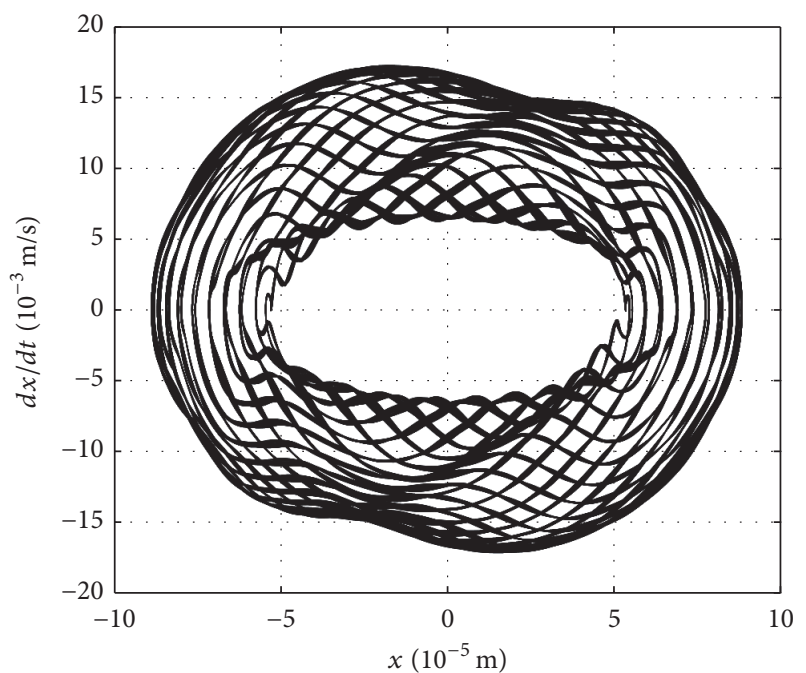

(c) Phase trajectory

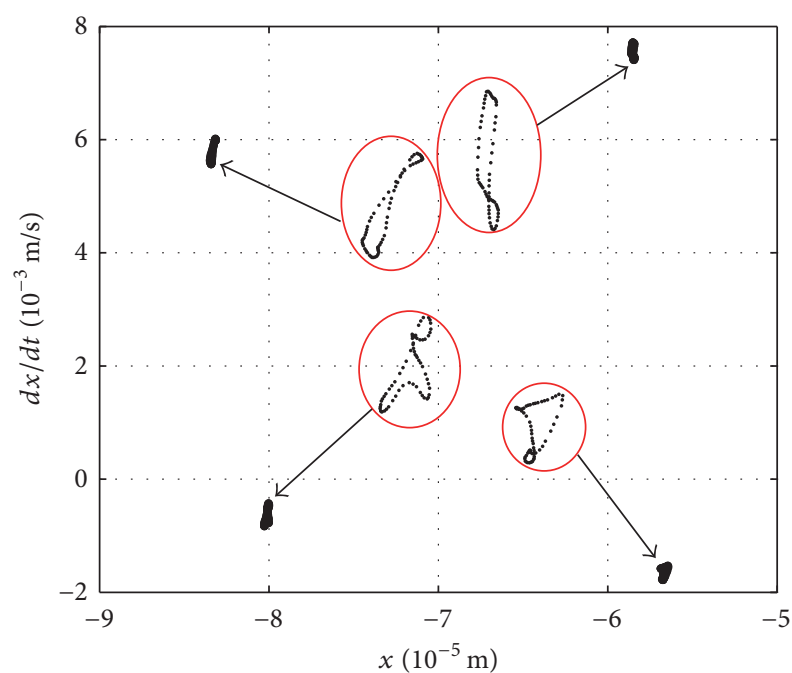

(d) Poincare map

Figure 16: Response analysis of disk 2: $\omega_{1}=170 \mathrm{rad} / \mathrm{s}$ and $\lambda=-1.65$. 


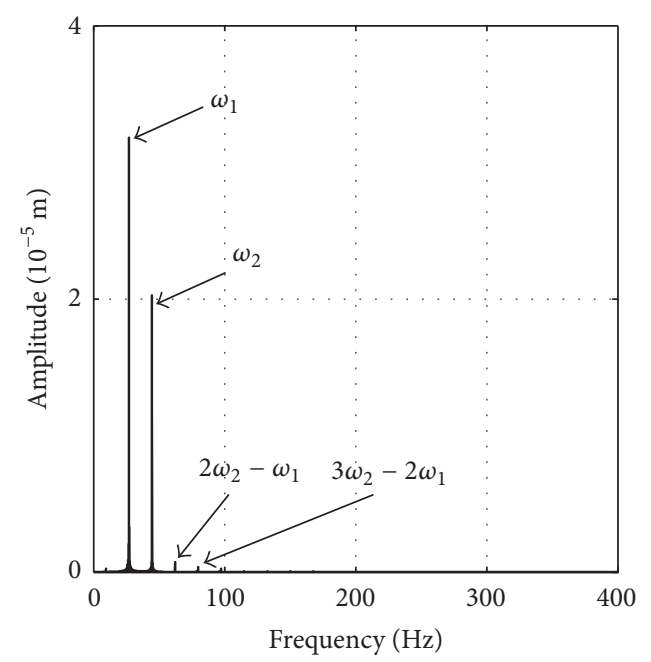

(a) Spectrogram

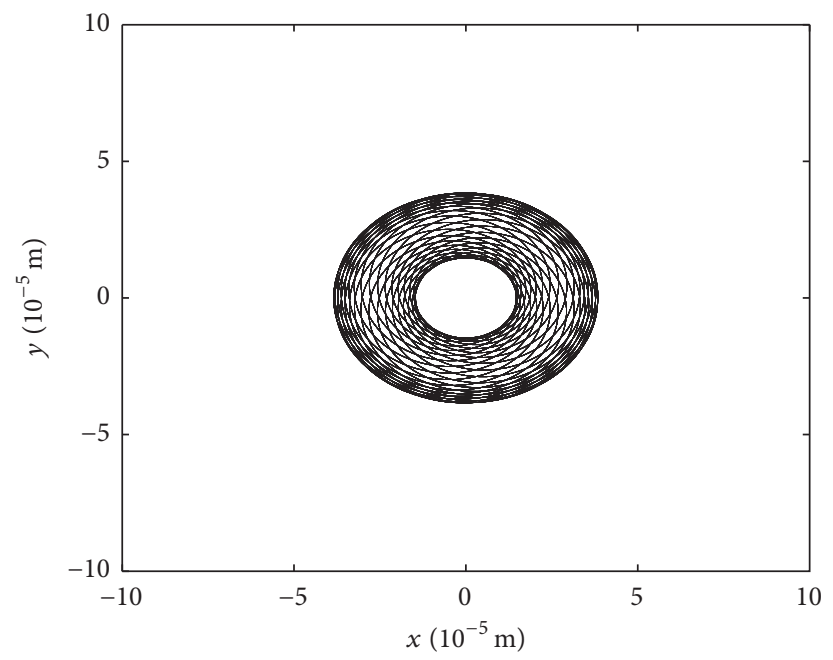

(b) Orbit

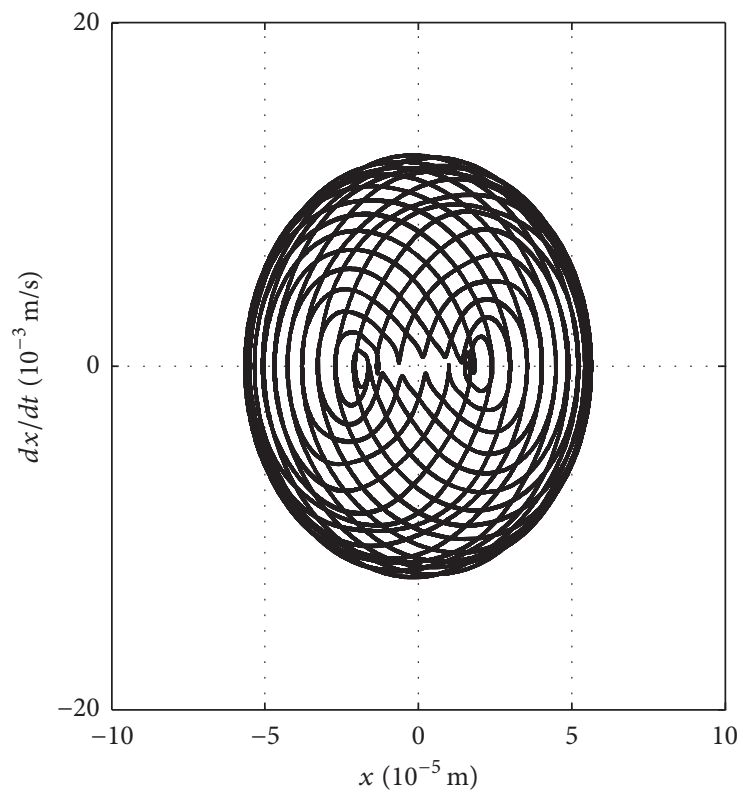

(c) Phase trajectory

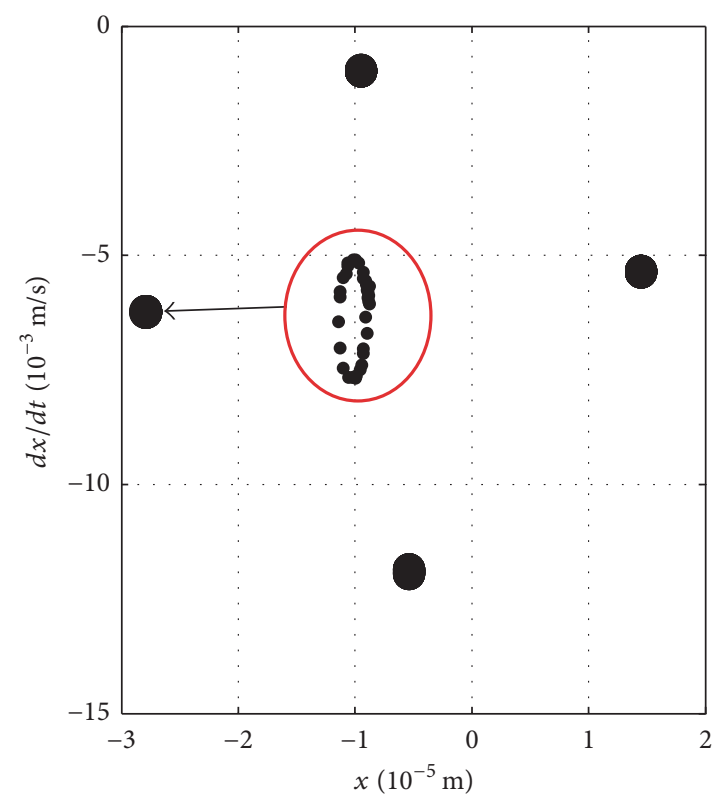

(d) Poincare map

FIGURE 17: Response analysis of disk 2: $\omega_{1}=170 \mathrm{rad} / \mathrm{s}$ and $\lambda=1.65$. 


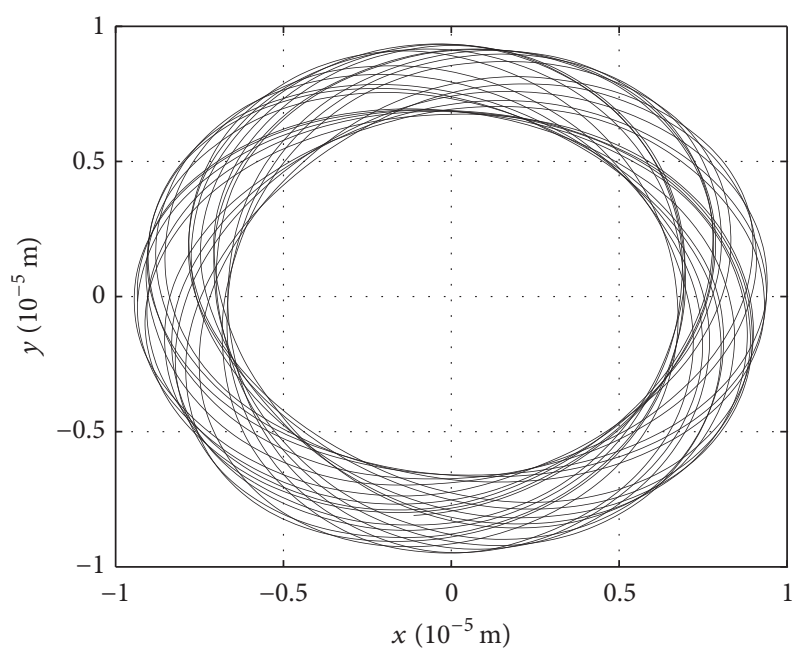

(a) Disk 2-numerical results

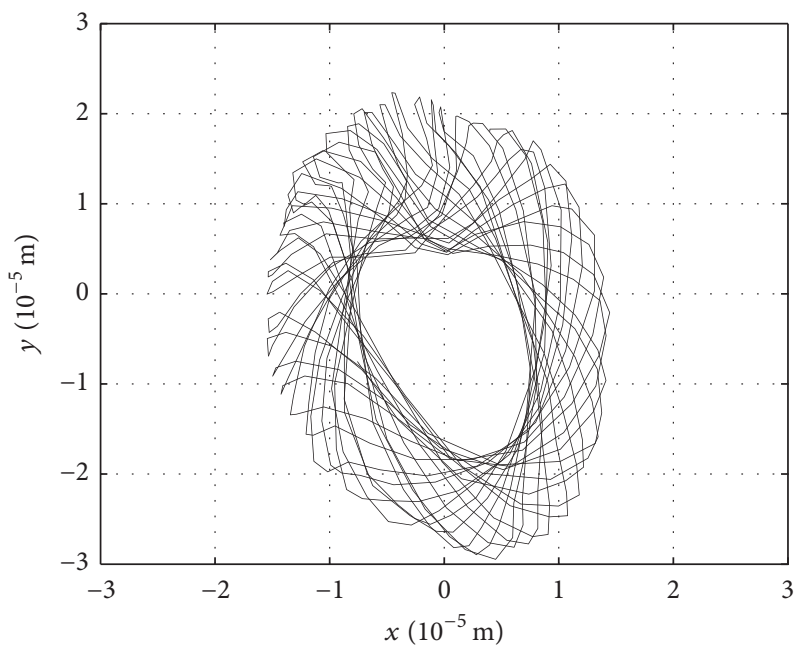

(b) Disk 2-experimental results

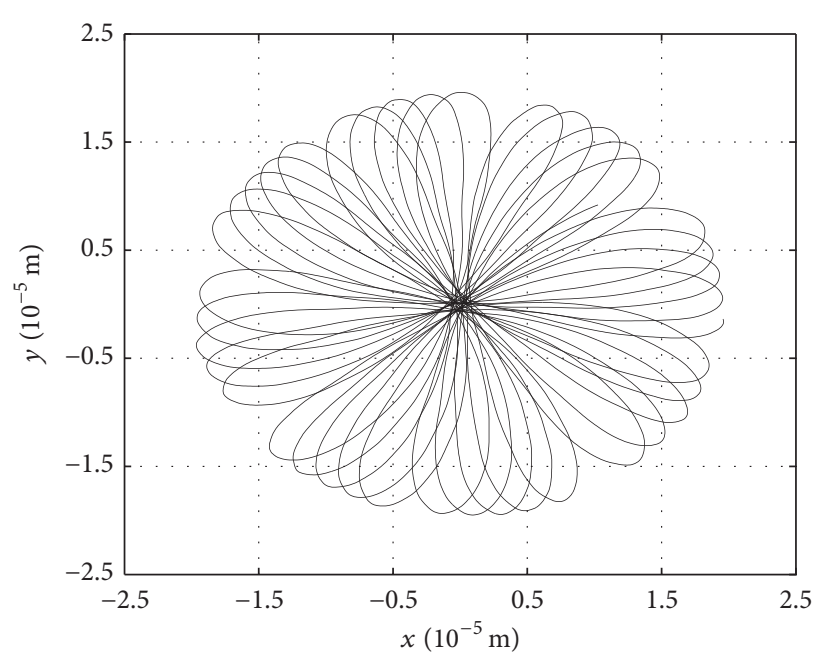

(c) Disk 4-numerical results

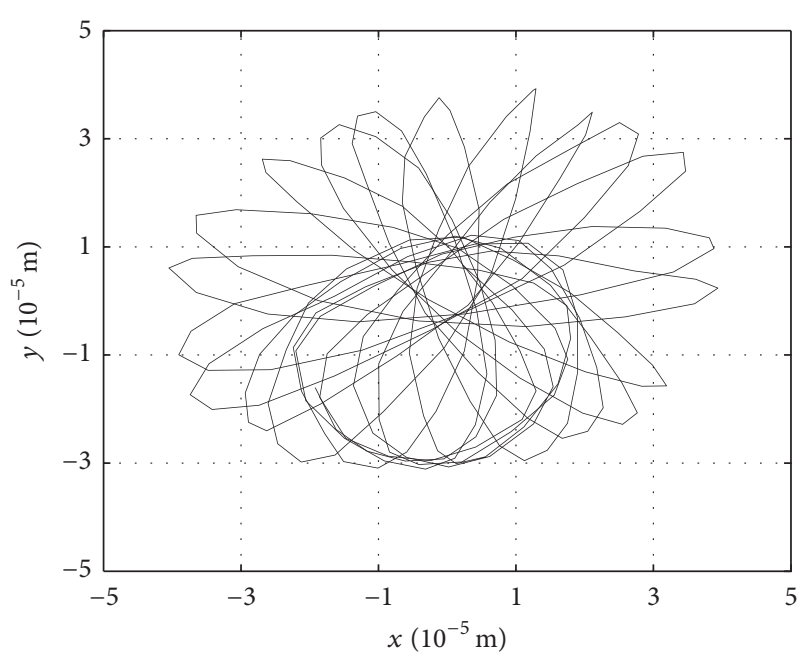

(d) Disk 4-experimental results

FIgURE 18: Orbit of disk 2 and disk 4 with $\omega_{1}=128 \mathrm{rad} / \mathrm{s}$ and $\lambda=-1.65$. 


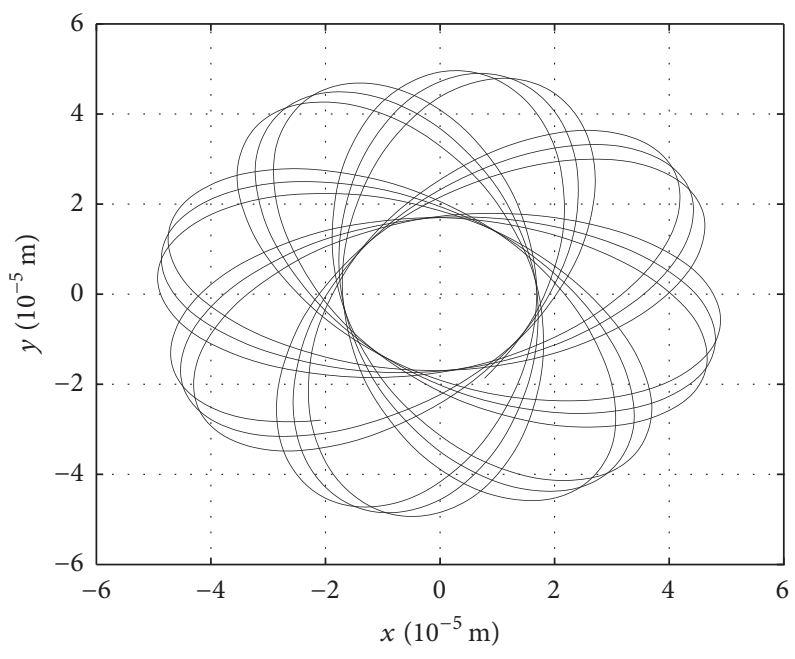

(a) Disk 2-numerical results

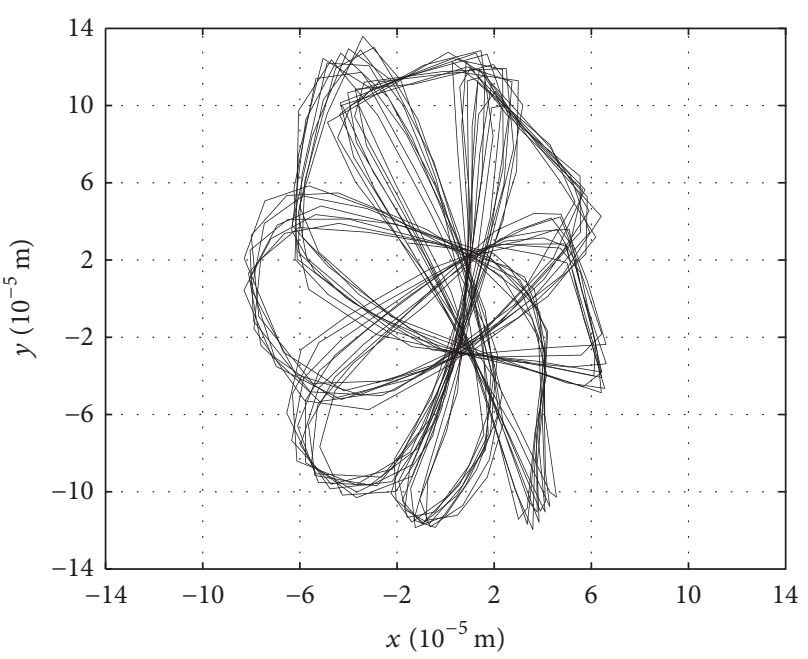

(b) Disk 2-experimental results

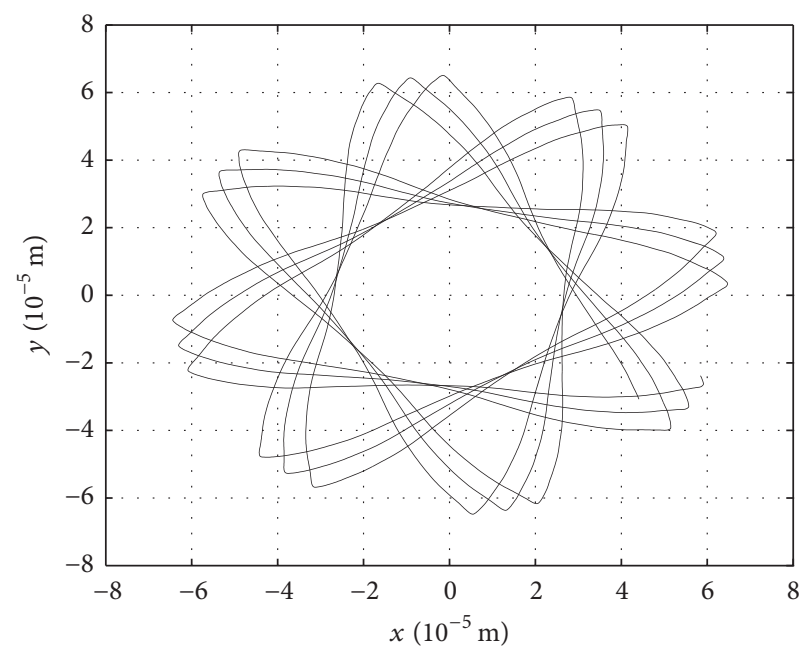

(c) Disk 4-numerical results

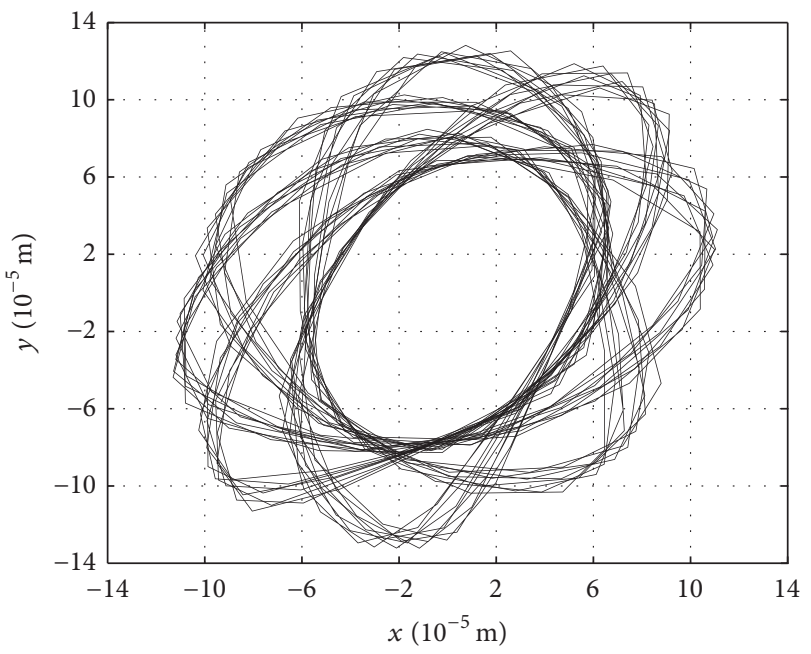

(d) Disk 4-experimental results

FIGURE 19: Orbit of disk 2 and disk 4 with $\omega_{1}=160 \mathrm{rad} / \mathrm{s}$ and $\lambda=-1.65$. 


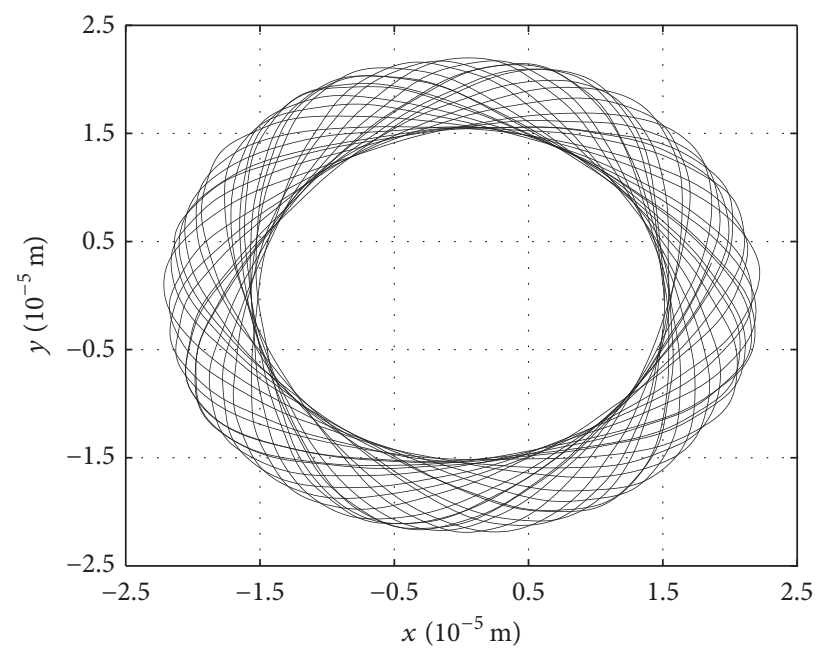

(a) Disk 2-numerical results

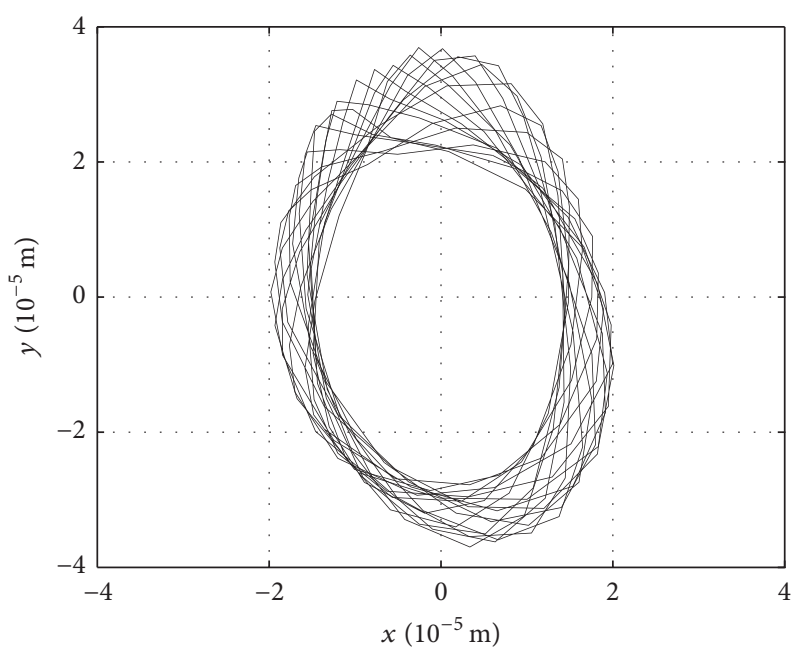

(b) Disk 2-experimental results

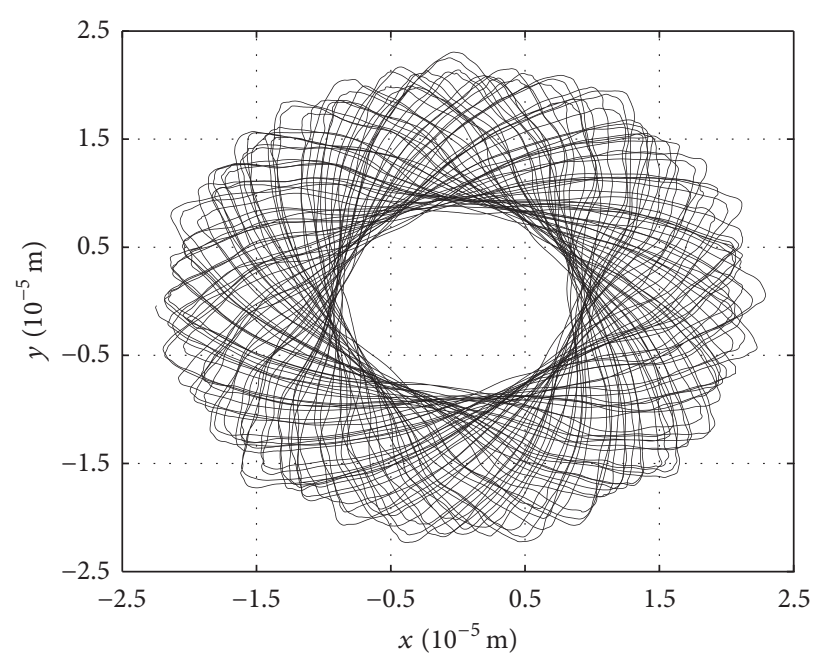

(c) Disk 4-numerical results

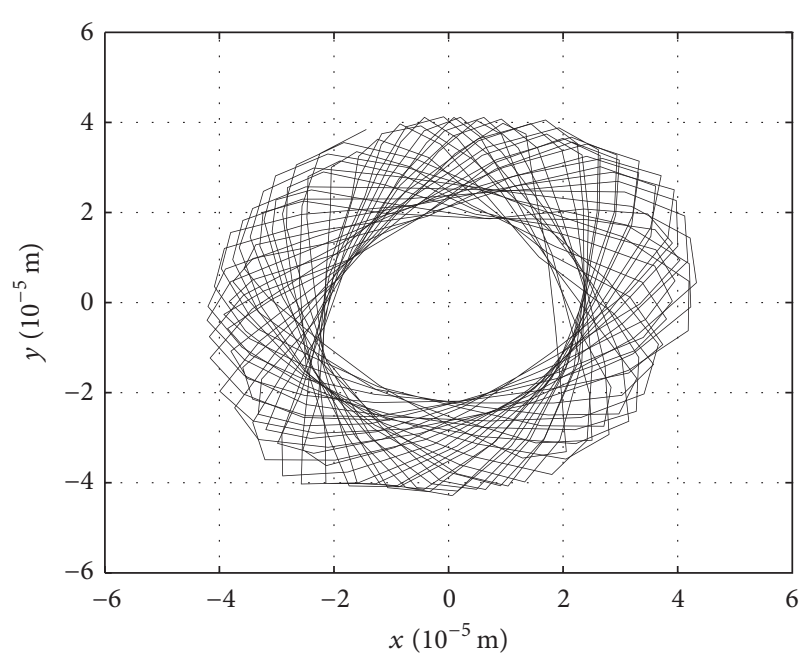

(d) Disk 4-experimental results

FIGURE 20: Orbit of disk 2 and disk 4 with $\omega_{1}=210 \mathrm{rad} / \mathrm{s}$ and $\lambda=-1.65$. 


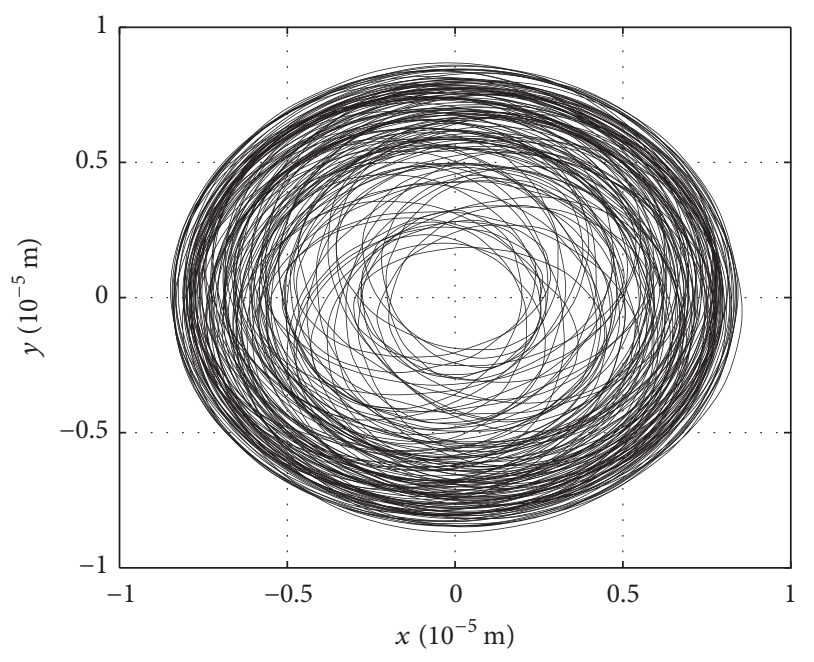

(a) Disk 2-numerical results

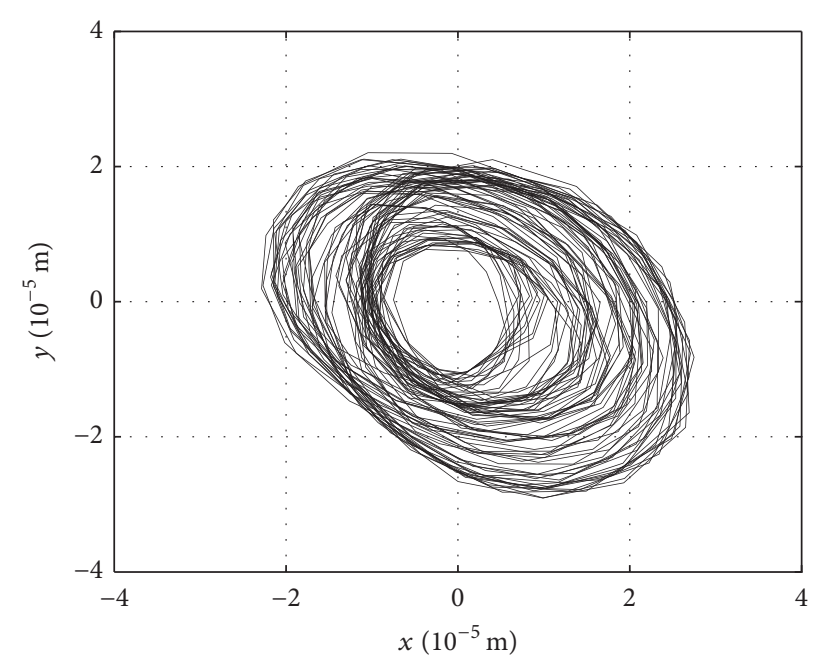

(b) Disk 2-experimental results

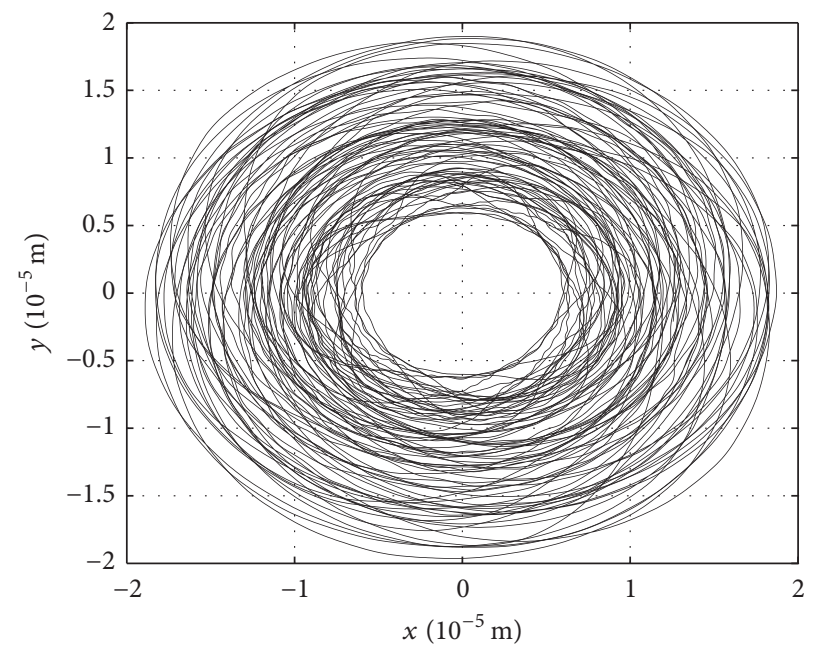

(c) Disk 4-numerical results

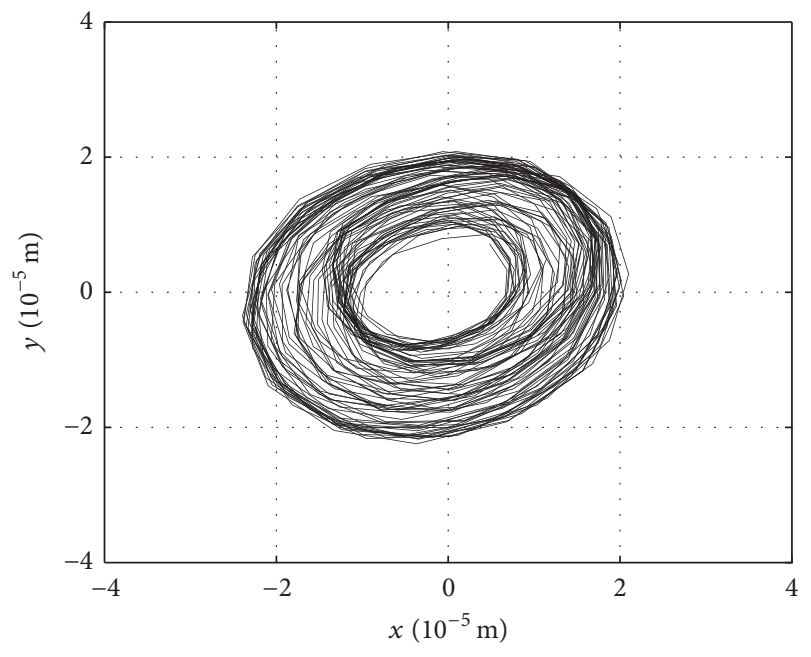

(d) Disk 4-experimental results

FIGURE 21: Orbit of disk 2 and disk 4 with $\omega_{1}=146 \mathrm{rad} / \mathrm{s}$ and $\lambda=1.65$. 


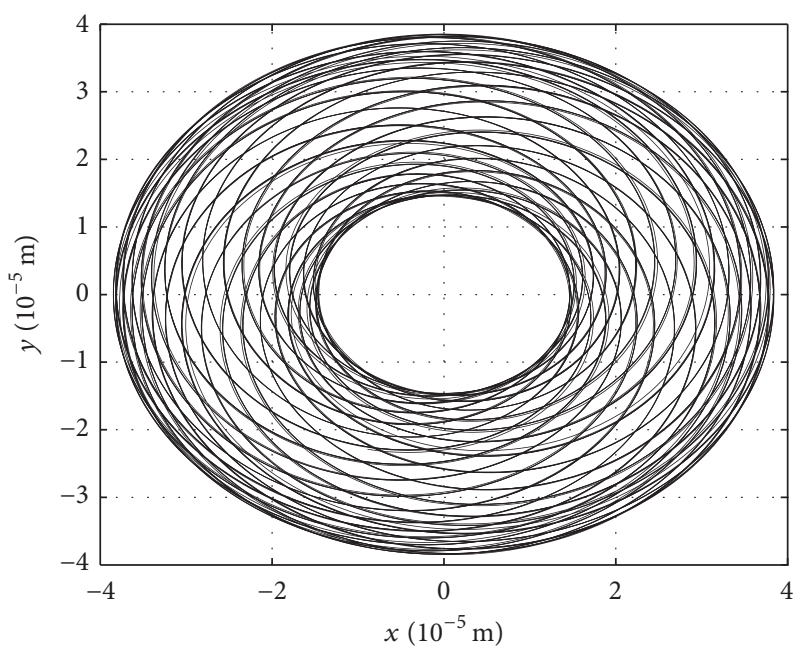

(a) Disk 2-numerical results

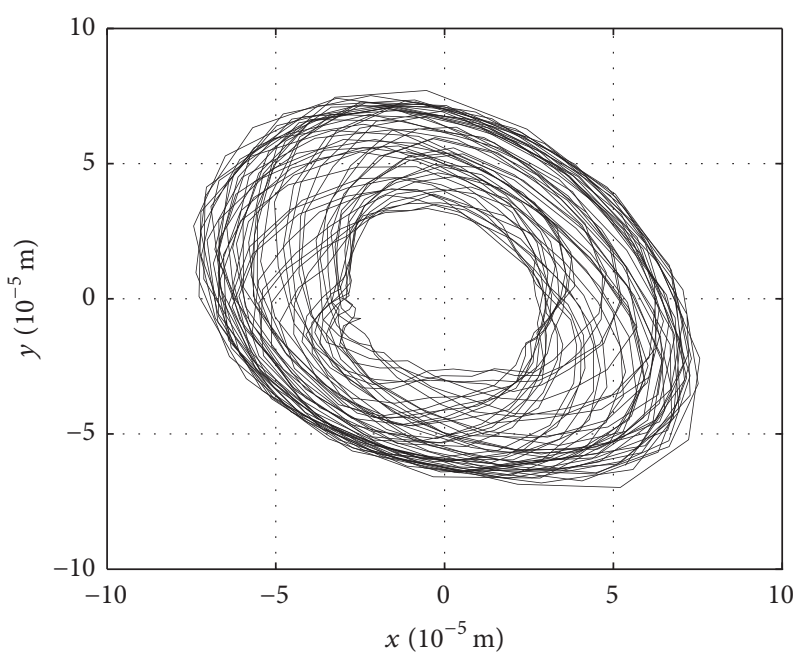

(b) Disk 2-experimental results

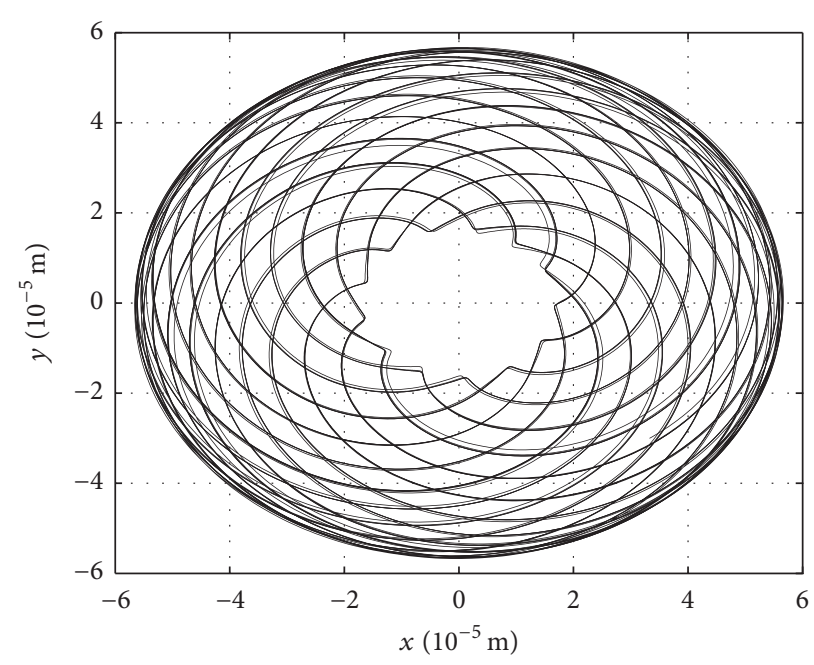

(c) Disk 4-numerical results

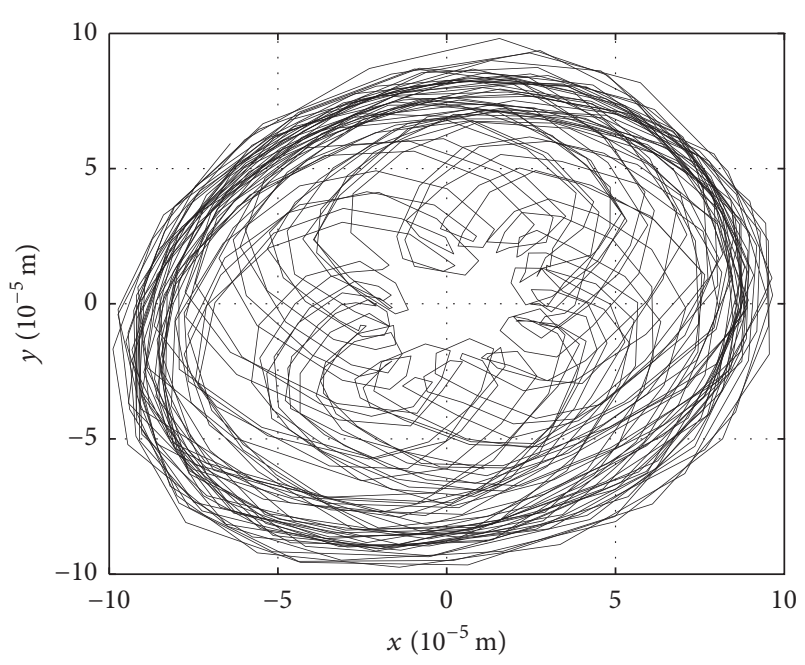

(d) Disk 4-experimental results

Figure 22: Orbit of disk 2 and disk 4 with $\omega_{1}=170 \mathrm{rad} / \mathrm{s}$ and $\lambda=1.65$. 
a counterrotating dual-rotor test rig for steady-state nonlinear response analysis. First, a finite element model of the rotor system is established without considering supports, from which the mass, stiffness, and gyroscopic matrices are obtained. Together with these matrices, the fixed interface modal synthesis method is applied to establish the nonlinear model of the rotor system in which the nonlinearities of SFD and intermediate bearing are considered. Subsequently, the Newmark-beta method is improved to solve the nonlinear equations of motion. Then dynamic characteristics of the rotor system are investigated.

Conclusions are listed as follows:

(1) The modeling method developed in this work is fast and efficient in establishing nonlinear model of complex rotor systems. Combining with the improved Newmark-beta method, nonlinear dynamic characteristics can be obtained accurately and efficiently.

(2) Due to the gyroscopic effect, critical speeds of the corotating rotor system are slightly higher than those of the counterrotating system.

(3) Due to coupling effect of the intermediate bearing, the dual unbalance excitation frequencies are dominant in the responses of inner and outer rotor for both the counter- and corotating systems. Besides, combination frequency components in the responses of inner and outer rotor are mainly caused by coupling effect of the intermediate bearing.

(4) Generally the rotors execute four period quasiperiodic motion around critical speeds. Between two critical speeds, the motion state of the rotor system can be quite different for the counter- and corotating cases. Around critical speeds, the amplitude of disk 2 is much larger for the counterrotating case comparing with that of the corotating case.

(5) The comparison between numerical and experimental results has shown great agreement, which proves validities of the modeling method and the numerical results.

\section{Conflicts of Interest}

The authors declare that there are no conflicts of interest regarding the publication of this paper.

\section{References}

[1] L. Ji, "Review and prospect of counter-rotating turbine," Aeroengine, vol. 32, no. 4, pp. 49-53, 2006 (Chinese).

[2] J. M. Vance and A. C. Royal, "High-speed rotor dynamics-an assessment of current technology for small turboshaft engines," Journal of Aircraft, vol. 12, no. 4, pp. 295-305, 1975.

[3] D. H. Hibner, "Dynamic response of viscous-damped multishaft jet engines," Journal of Aircraft, vol. 12, no. 4, pp. 305-312, 1975.

[4] K. Gupta, K. D. Gupta, and K. Athre, "Unbalance response of a dual rotor system: theory and experiment," Journal of Vibration and Acoustics, vol. 115, no. 4, pp. 427-435, 1993.
[5] G. Ferraris, V. Maisonneuve, and M. Lalanne, "Prediction of the dynamic behavior of non-symmetric coaxial co- or counterrotating rotors," Journal of Sound and Vibration, vol. 195, no. 4, pp. 649-666, 1996.

[6] M. Lalanne and G. Ferraris, Rotordynamics Prediction in Engineering, John Wiley \& Sons, 1990.

[7] X. Hu, G.-H. Luo, and D.-P. Gao, "Numerical analysis and experiment of counter-rotating dual-rotor's steady-state response," Journal of Aerospace Power, vol. 22, no. 7, pp. 10441049, 2007 (Chinese).

[8] G. H. Luo, X. Hu, and X. Yang, "Nonlinear dynamic performance analysis of counter-rotating dual-rotor system," Journal of Vibration Engineering, vol. 22, no. 3, pp. 268-273, 2009 (Chinese).

[9] G. Feng, C. Yue, and L. Zhang, "Dynamic analysis of a two-spool engine with counter rotating rotors," Aeroengine, vol. 19, no. 5, pp. 43-48, 1993 (Chinese).

[10] H.-W. D. Chiang, C.-N. Hsu, and S.-H. Tu, "Rotor-bearing analysis for turbomachinery single- and dual-rotor systems," Journal of Propulsion and Power, vol. 20, no. 6, pp. 1096-1104, 2004.

[11] M. Guskov, J.-J. Sinou, F. Thouverez, and O. S. Naraikin, "Experimental and numerical investigations of a dual-shaft test rig with intershaft bearing," International Journal of Rotating Machinery, vol. 2007, Article ID 75762, 12 pages, 2007.

[12] X. Hu, G.-H. Luo, and D.-P. Gao, "Performance analysis of aeroengine intershaft bearing," Journal of Aerospace Power, vol. 22, no. 3, pp. 439-443, 2007 (Chinese).

[13] P. M. Hai and P. Bonello, "An impulsive receptance technique for the time domain computation of the vibration of a whole aero-engine model with nonlinear bearings," Journal of Sound and Vibration, vol. 318, no. 3, pp. 592-605, 2008.

[14] P. Bonello and P. M. Hai, "A receptance harmonic balance technique for the computation of the vibration of a whole aeroengine model with nonlinear bearings," Journal of Sound and Vibration, vol. 324, no. 1-2, pp. 221-242, 2009.

[15] P. Bonello and P. M. Hai, "Computational studies of the unbalance response of a whole aero-engine model with squeeze-film bearings," Journal of Engineering for Gas Turbines and Power, vol. 132, no. 3, Article ID 032504, 2010.

[16] P. M. Hai and P. Bonello, "A computational parametric analysis of the vibration of a three-spool aero-engine under multifrequency unbalance excitation," Journal of Engineering for Gas Turbines and Power, vol. 133, no. 7, Article ID 072504, 9 pages, 2011.

[17] J. I. Inayat-Hussain, "Bifurcations in the response of a flexible rotor in squeeze-film dampers with retainer springs," Chaos, Solitons and Fractals, vol. 39, no. 2, pp. 519-532, 2009.

[18] B. Mevel and J. L. Guyader, "Experiments on routes to chaos in ball bearings," Journal of Sound and Vibration, vol. 318, no. 3, pp. 549-564, 2008. 


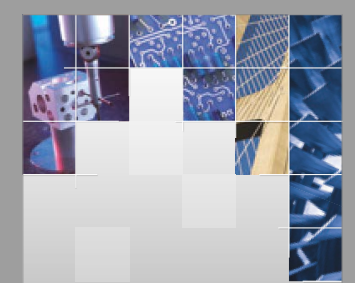

\section{Enfincering}
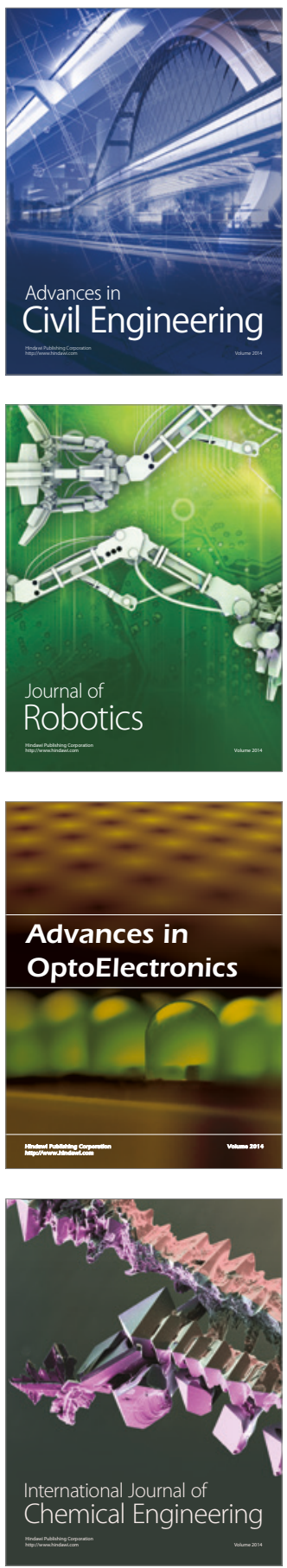

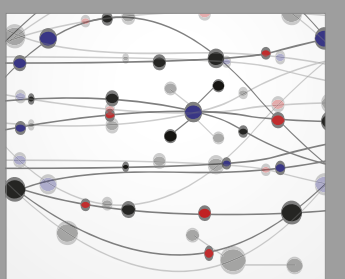

The Scientific World Journal

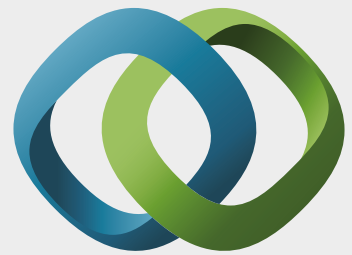

\section{Hindawi}

Submit your manuscripts at

https://www.hindawi.com
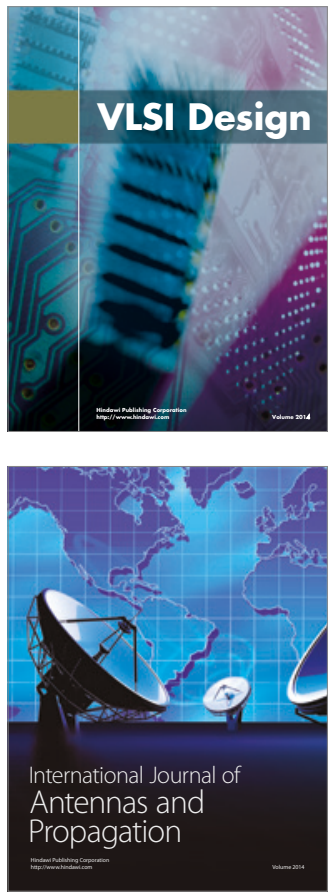

\section{Rotating}

Machinery
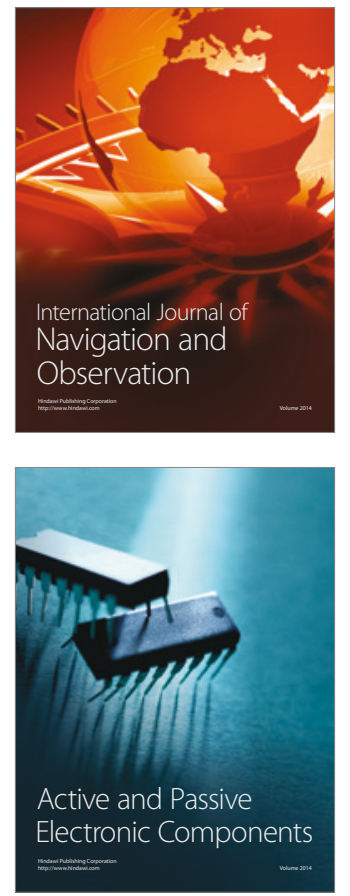
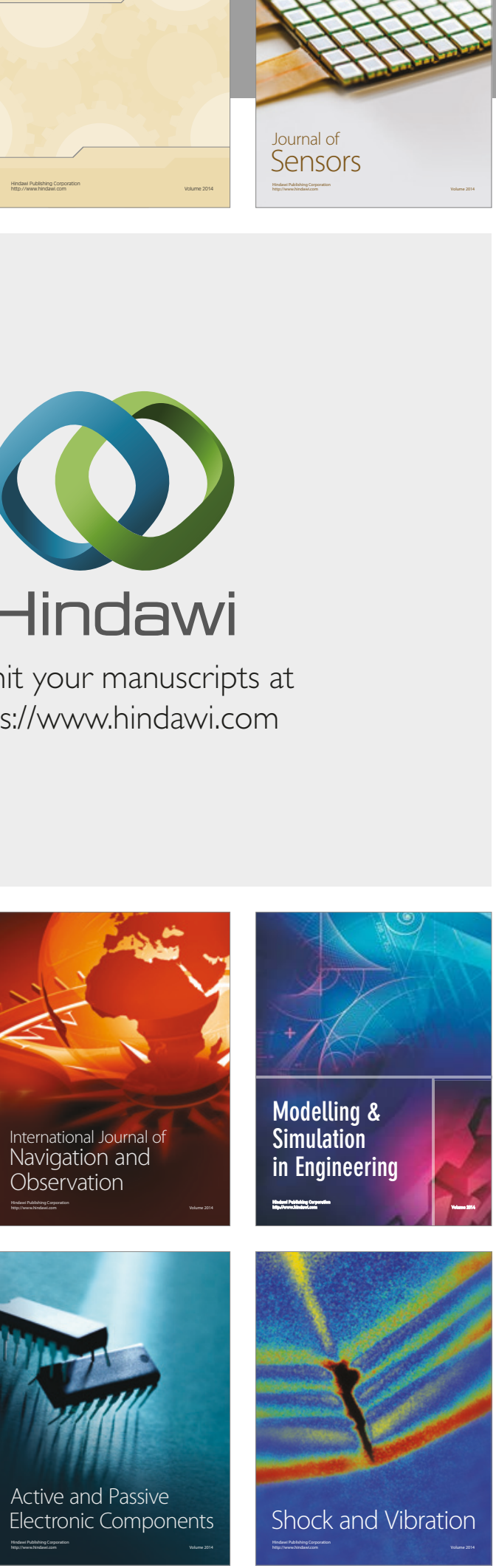
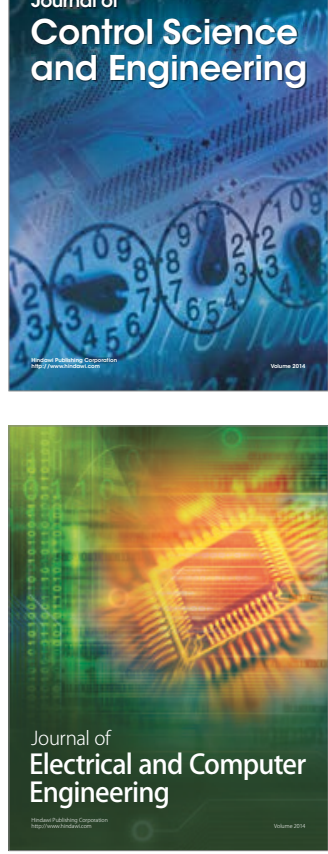

Distributed

Journal of

Control Science

and Engineering
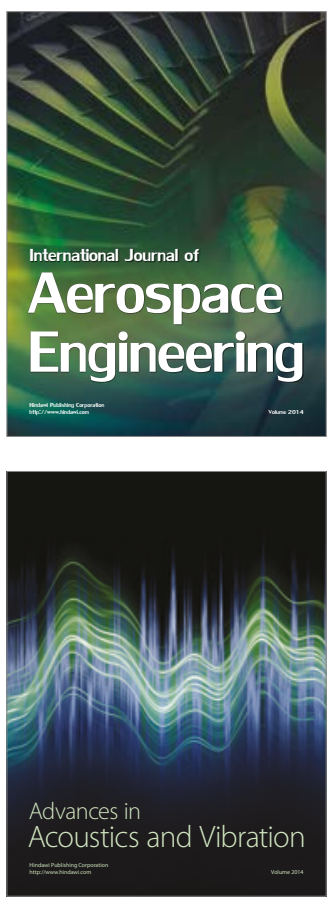

Sensor Networks 